\title{
Historia de la inspección y auscultación de estructuras en el Laboratorio Central
}

\section{History of Inspection and Monitoring of Structures in Laboratorio Central}

\author{
Ismael Carpintero García ${ }^{\mathrm{a}}$, Jorge Rueda Puerta ${ }^{\mathrm{b}}$ \\ ${ }^{a, b}$ Ingeniero de Caminos, Canales y Puertos. Laboratorio Central de Estructuras y Materiales (CEDEX) \\ a Jefe del Área de Estudios y Auscultación de Estructuras \\ ${ }^{b}$ Coordinador Técnico-Cientifico del Área de Estudios y Auscultación de Estructuras
}

Recibido el 18 de diciembre de 2020; aceptado el 30 de marzo de 2021

\begin{abstract}
RESUMEN
El artículo busca ilustrar el origen de las actividades de inspección, auscultación y evaluación de estructuras en el Laboratorio Central del CEDEX, creado inicialmente, en 1898, como Laboratorio Central de Ensayo de Materiales de Construcción de la antigua Escuela Especial de Ingenieros de Caminos, Canales y Puertos del Retiro. Aunque fue concebido, en principio, para el ensayo de materiales de construcción, muy pronto, ya en la primera década del siglo XX, comenzaron a realizarse también ensayos sobre productos de construcción. Posteriormente, de manera progresiva y de la mano de algunos de los más insignes ingenieros españoles del siglo XX, se pasó a realizar trabajos de investigación sobre elementos estructurales y modelos reducidos, pruebas de carga sobre obras construidas y, finalmente, inspecciones y auscultaciones de estructuras. El Laboratorio Central ha actuado como perito en procesos judiciales desde las primeras décadas del siglo XX, y ha sido precursor de buena parte de las tecnologías que más tarde se han ido utilizando en el sector de la inspección, auscultación y evaluación de estructuras (fotoelasticidad, ensayos sobre modelos reducidos, ensayos de caracterización en obra de materiales, pruebas de carga estáticas y dinámicas, inspecciones con medios especiales de acceso, etc). Ha cumplido de esta forma una de las máximas que guían su actividad y que se recogen en sus estatutos: la misión de impulsar la transferencia tecnológica a la sociedad para abrir nuevos caminos a la ingeniería.
\end{abstract}

PALABRAS CLAVE: Inspección y auscultación de estructuras, Laboratorio Central, CEDEX.

(C) 2021 Asociación Española de Ingeniería Estructural (ACHE). Publicado por Cinter Divulgación Técnica S.L. Todos los derechos reservados.

\section{ABSTRACT}

The article seeks to illustrate the origin of the inspection, monitoring and evaluation of structures in the Central Laboratory of CEDEX. It was initially created in 1898 as the Central Laboratory for Testing of Construction Materials of the old Escuela Especial de Ingenieros de Caminos, Canales y Puertos at Retiro Park. Although it was conceived for the testing of construction materials, tests on construction products also began as early as the first decade of the 20th century. Later, progressively and hand in hand with some of the most distinguished Spanish engineers of the 20th century, research work began in structural elements and reduced models, load tests on built works and, finally, inspections and monitoring of structures. The Central Laboratory has acted as an expert in legal proceedings since the first decades of the 20th century, and it has been the forerunner of a good part of the technologies that have subsequently been used in the field of inspection, monitoring and evaluation of structures (photoelasticity, tests on reduced models, characterization tests of materials on site, static and dynamic load tests, inspections with special means of access, etc). In this way, it has fulfilled one of the principles that guide its activity and that are included in its statutes: the mission of promoting technology transfer to society to open new paths to engineering.

KEYWORDS: Inspection and monitoring of structures, Laboratorio Central, CEDEX.

(C) 2021 Asociación Española de Ingeniería Estructural (ACHE). Published by Cinter Divulgación Técnica S.L. All rights reserved.

* Persona de contacto / Corresponding author:

Correo-e / email: ismael.carpintero@cedex.es (Ismael Carpintero García).

Cómo citar este artículo: Carpintero García, I., Rueda Puerta, J. (2021) Historia de la inspección y auscultación de estructuras en el Laboratorio Central, Hormigón y Acero, 72(294-295), 7-22, https://doi.org/10.33586/hya.2021.3060 
1.

\section{BREVE RESEÑA HISTÓRICA DE LAS INSTALACIONES Y ORGANIZACIÓN DEL LABORATORIO CENTRAL DEL CEDEX}

El actual Laboratorio Central de Estructuras y Materiales ${ }^{1}$ del CEDEX hunde sus raíces en 1898, cuando se crea por Real Decreto de la Reina Regente el primer laboratorio central de materiales adscrito a la Escuela Especial de Ingenieros de Caminos, Canales y Puertos, con el objetivo de (...) estudiar las propiedades y principalmente la resistencia de los materiales que con este objeto se le entreguen por el Estado, las Corporaciones y por los particulares (...). Aunque su creación vino a atender las necesidades que planteaba el sector de la construcción con la aparición de nuevos materiales en el siglo XIX ${ }^{2}$, en realidad el Laboratorio Central no solo se ha dedicado al ensayo de materiales, sino que ya desde sus inicios comenzaron a ensayarse también equipos industriales para emitir certificados de idoneidad, tarea que se recogía en su primer reglamento.

El Laboratorio Central se instaló inicialmente en las dependencias de la planta inferior de la antigua Escuela Especial de Ingenieros de Caminos, Canales y Puertos, situada en el cerrillo de San Blas, junto al parque del Retiro (figura 1). A tal efecto, los dos patios interiores del edificio se cubrieron bajo sendas cubiertas metálicas prefabricadas traídas expresamente desde Baviera ${ }^{3}$.
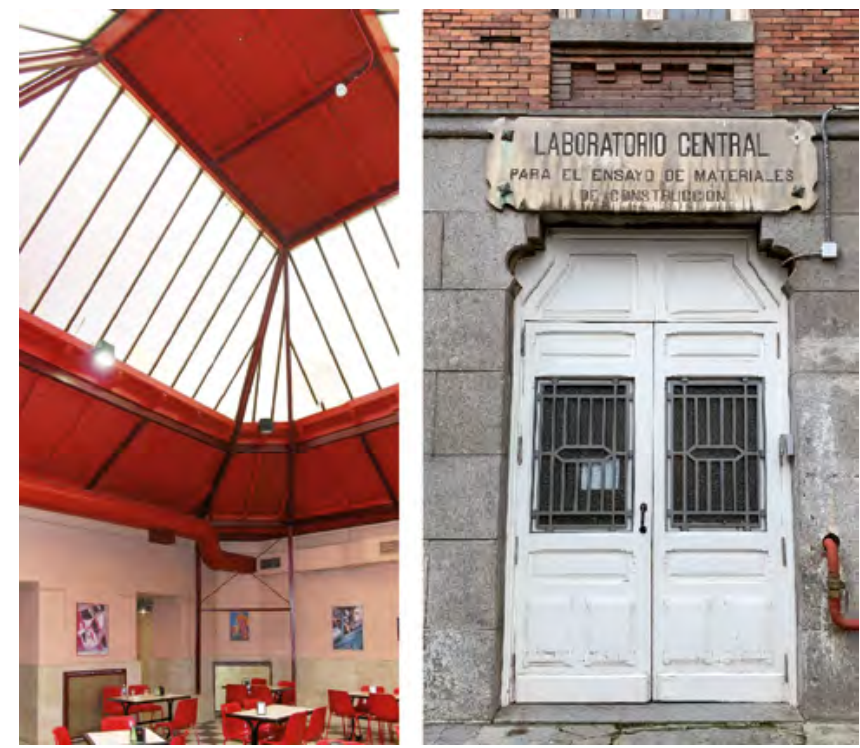

Figura 1. Espacio del Laboratorio Central en la Escuela del Retiro.

1 En origen se creó con el nombre de Laboratorio Central para el Ensayo de Materiales de Construcción.

$2 \mathrm{El}$ acero estructural de los hornos de pudelado, Martin-Siemens y Bessemer; el hormigón armado de la época de las patentes; los materiales bituminosos para firmes de carreteras, etc. En 1896 hubo una propuesta de enmienda a la Ley de Presupuestos para crear un laboratorio central (...) poniéndonos a la altura de las demás naciones y evitando el caso vergonzoso de que tengamos que recurrir al extranjero, como ha ocurrido, para hacer las pruebas de las traviesas metálicas del ferrocarril de Calatayud-Teruel-Sagunto (...)

3 En 1898 la industria siderúrgica española estaba iniciando su camino con cierto retraso respecto a otros países europeos, por lo que, si bien los forjados del edificio se construyeron con perfiles laminados de ala estrecha de DURO \& FELGUERA, la estructura de cubrición de los patios interiores y las cerchas de la cubierta se fabricaron en la STAHLWERK ANNAHÜTTE (actualmente integrada en el grupo MAX AICHER GMBH \& CO), cuyo origen se remonta a 1537, situada entonces en el Imperio Alemán, muy cerca de Salzburgo
Paulatinamente, este laboratorio original fue ampliando sus competencias a medida que iban apareciendo nuevas necesidades, obligando a ampliar y especializar las instalaciones que en origen se encontraban en el interior de la antigua Escuela de Caminos, y a incorporar otros edificios aledaños en los que colocar los nuevos laboratorios.

De esta forma, en 1917 se construyó en un pequeño edificio anexo el Laboratorio de Electro-Mecánica y el Laboratorio de Alumnos (donde durante varias reordenaciones se alojarían también los Laboratorios de Ingeniería Sanitaria y Termotecnia); y en 1942 se construyó el Laboratorio de Hidráulica en el pequeño edificio que actualmente acoge la Secretaría del CEDEX. Dicho Laboratorio de Hidráulica fue el antecesor del Centro de Estudios Hidrográficos, que se trasladó a su localización actual en 1963. En 1943 las necesidades de espacio obligaron a erigir un nuevo edificio detrás de la antigua Escuela de Ingenieros de Caminos, donde se ha ubicado desde entonces el Laboratorio Central.

Poco a poco, el desarrollo de la ingeniería española fue requiriendo nuevas instalaciones que inicialmente se ubicaban en el citado edificio del Laboratorio Central, y que paulatinamente demandaban nuevos espacios. Así, en 1944 se creó el Laboratorio de Transporte y Mecánica del Suelo, que en 1954 se trasladó a un nuevo edificio en el mismo cerrillo de San Blas. Este Laboratorio fue el germen, en 1985, del Laboratorio de Geotecnia, y del Centro de Estudios de Carreteras (actual Centro de Estudios del Transporte), que entonces se trasladó a sus instalaciones de El Goloso. El Laboratorio de Puertos, creado en 1951, se trasladó ya como Centro de Estudios de Puertos y Costas a sus instalaciones actuales en 1980.

La promulgación de la Ley de Enseñanzas Técnicas de 1957 supuso que la Escuela pasara de depender del Ministerio de Obras Públicas al de Educación. Sin embargo, los Laboratorios fueron asignados al Ministerio de Obras Públicas, integrándose todos ellos en un solo Organismo de nueva creación (decreto de 23 de agosto de 1957), el Centro de Estudios y Experimentación de Obras Públicas (CEDEX), con la encomienda de llevar a cabo de manera generalizada el estudio y experimentación de todas las técnicas relacionadas con la obra pública.

En 1986 se integró en el CEDEX el Centro de Estudios Históricos de Obras Públicas y Urbanismo (creado tres años antes); y en 1985, fruto de una profunda reorganización, se creó el Centro de Estudios de Técnicas Aplicadas, que agrupó e integró en el CEDEX distintos servicios y centros de apoyo transversal a la ingeniería (el Gabinete de Cálculo, el Centro Bibliográfico y el Gabinete de Aplicaciones Nucleares a las Obras Públicas, el Servicio de Técnicas Físicas, el Departamento de Formación y el de Medio Ambiente). Este Centro se trasladó a su actual edificio en 1990.

En 1980 se integró en el Laboratorio Central el Laboratorio del Instituto Nacional de Calidad de la Edificación (INCE, creado en 1972), y en el 2000 el Laboratorio de la Dirección General de la Vivienda, la Arquitectura y el Urbanismo ${ }^{4}$. El úl-

4 En realidad, en 1980 se publicó en el BOE la Orden de creación del Instituto Tecnológico de las Obras Públicas (resultado de integrar el Laboratorio del INCE en el CEDEX), pero no llegó a llevarse a efecto. En el 2000, una vez transferidos a las comunidades autónomas los centros regionales del INCE, se integró en el Laboratorio Central del CEDEX el Laboratorio de Madrid del INCE (que se había transformado en el Laboratorio de la Dirección General de la Vivienda, la Arquitectura y el Urbanismo). 

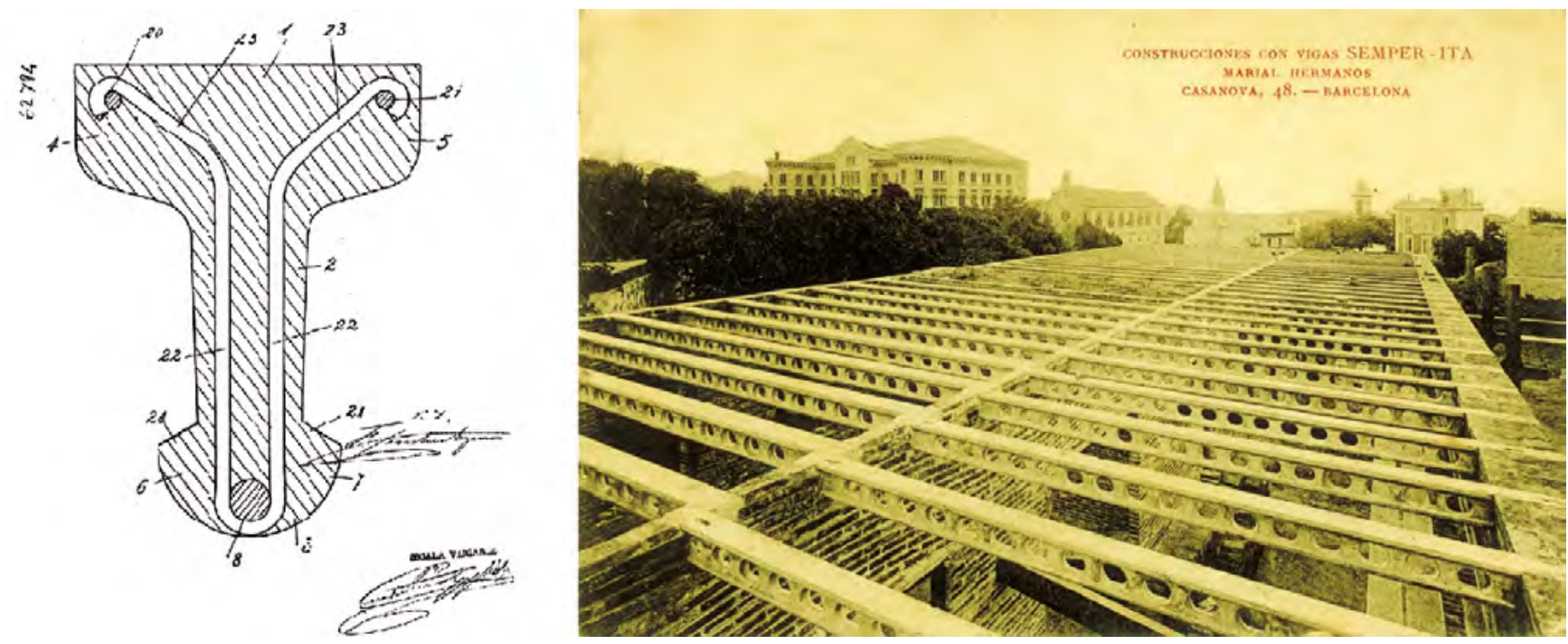

Figura 2. Viguetas de la patente SEMPER-ITA 5 .

timo centro especializado creado en el CEDEX fue el Laboratorio de Interoperabilidad Ferroviaria. Su origen se remonta a 1994, adscrito inicialmente al Centro de Estudios de Técnicas Aplicadas y, posteriormente, al Centro de Estudios del Transporte, y que tomó cuerpo como Laboratorio independiente en 2001, cuando se trasladó a sus instalaciones actuales.

\section{2.}

\section{DE LOS ENSAYOS DE MATERIALES A LOS ENSAYOS DE MODELOS}

Durante las primeras décadas desde su creación, en el Laboratorio Central se realizaron ensayos físicos y químicos tanto de materiales y productos de construcción (cementos portland y naturales, cales, áridos, agua, alquitranes y betunes, madera, carbón, creosota, piedra, ladrillos, baldosas), como de materiales y productos para el sector industrial (motores, correas de transmisión, neumáticos, grasas o cables). También durante estos años se llevó a cabo la certificación de productos mediante ensayos de validación (voltímetros, amperímetros, vatímetros, contadores eléctricos).

Si bien por número de expedientes la actividad más frecuente en el Laboratorio Central fueron claramente los ensayos de materiales, ya en 1907 comenzaron a realizarse ensayos mecánicos sobre tubos (de gres, hierro, plomo o acero). El ensayo mecánico fundamental fue el de la resistencia del tubo a la presión interior. Este tipo de ensayos se realizó de forma profusa hasta la década de los años 30 del siglo XX.

El primer elemento estructural objeto de ensayo fue un pilote de hormigón armado ensayado a flexión en tres puntos en 1915. En esa misma década se realizaron también ensayos mecánicos de resistencia a flexión sobre carriles reutilizados del ferrocarril de Tetuán a Tánger, o sobre las nuevas viguetas de hormigón armado de la patente SEMPER-ITA de Barcelona (figura 2).

5 Para más información sobre esta patente se puede consultar [1].
El primer responsable de este tipo de ensayos mecánicos fue Juan Alonso Millán, quien fuera Jefe del Laboratorio a las órdenes del entonces Director del Laboratorio y de la Escuela, Rogelio de Inchaurrandieta. Estos ensayos los realizaban ingenieros jóvenes (o estudiantes de la Escuela) como Bienvenido Oliver y Román. En 1911 pasó a encargarse de ellos Andrés Morán y Arroyo ${ }^{6}$. Durante los siguientes años la mayor parte de los ensayos mecánicos sobre tubos y elementos estructurales los dirigirian primero P.F. Quintana, y posteriormente Juan Lázaro Urra. Entre 1925 y 1929, Alfonso Peña Boeuf se encargaba de realizar los ensayos mecánicos en el Laboratorio, mientras que el citado Andrés Morán se concentraba en la realización de los ensayos químicos.

Además de los ensayos sistemáticos sobre materiales de construcción, y de los ensayos mecánicos sobre tubos y viguetas, empezaron a llegar también nuevos materiales de construcción al Laboratorio Central para la realización de ensayos que certificasen sus prestaciones. Así, en 1914 se realizaron ensayos de caracterización mecánica de una pizarra artificial de nombre comercial "Uralita"; y en 1927 se realizaron ensayos para analizar la eficacia de algunos productos de Sika (figura 3).

Durante los años 30, hasta la Guerra Civil, se realizaron numerosos ensayos de viguetas a flexión pura encargados por Carlos Fernández Casado en representación de Huarte, y llevados a cabo por P. Becerril. En 1934 se creó la Sección de Fotoelasticidad ${ }^{7}$ (tecnología que se mantendría en el Laboratorio Central hasta los años 70), lo que supuso un salto cualitativo en el ámbito de actuación del Laboratorio: pasar del paradigma de laboratorio de referencia para el ensayo de materiales al de organismo de investigación.

6 Quien actuó como Jefe del Laboratorio desde 1929, aunque fue en 1934 cuando se convirtió en el primer Director del mismo como cargo independiente de la Escuela.

7 Técnica que básicamente consistía en construir un modelo con plexiglás sobre el que se aplicaban cargas mediante actuadores. Las deformaciones se evaluaban analizando la dispersión de la luz en el material, y con el empleo de comparadores y extensómetros, y, posteriormente, con bandas extensométricas. 

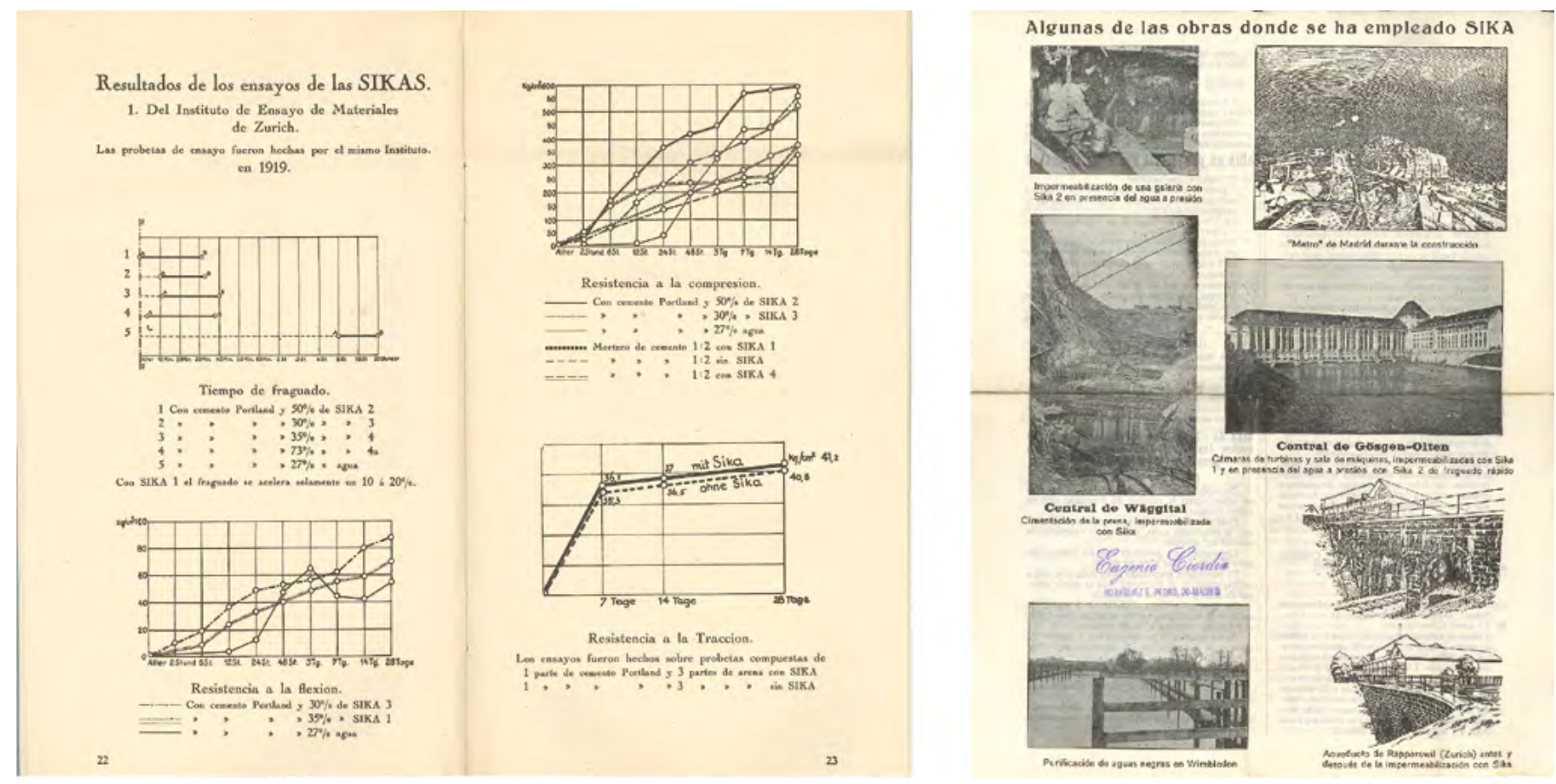

Figura 3. Folleto informativo de SIKA (1927).

La Guerra Civil produjo una fuerte ralentización de la actividad del Laboratorio, aunque se siguieron realizando ensayos sobre materiales hasta el asedio de Madrid por el bando Nacional. En esa época los encargos se centraban en ensayos sobre materiales empleados en infraestructuras de reconstrucción y defensa, encargados por organismos militares. El único periodo en el que el Laboratorio interrumpió sus trabajos fue de octubre de 1938 a mayo de 1939 .

\section{3.}

\section{TORROJA EN EL LABORATORIO CENTRAL}

La llegada en 1940 de Eduardo Torroja a la dirección del Laboratorio Central supuso un impulso a la investigación interna: se desarrollaron los primeros trabajos de la Sección de Fotoelasticidad que se había creado antes de la Guerra Civil, con la publicación de varias monografías sobre los principios que regían el sistema y la preparación de prototipos para la evaluación de modelos; y se iniciaron los ensayos con modelos reducidos de estructuras y elementos estructurales (figura 4), que tanta importancia adquirieron en las décadas siguientes. También se continuó con el ensayo de nuevos materiales, como distintas adiciones para el hormigón, o colas para el pegado de tablillas y virutas de madera para construir tableros de aglomerado (Melocol).

Para hacer frente a los nuevos retos del organismo, Eduardo Torroja promovió dos cambios trascendentales en la evolución del Laboratorio: una reorganización de la estructura interna y la construcción de un nuevo edificio que diese cabida a las necesidades futuras (figura 5). Cabe destacar la visión y empuje de Torroja, quien, en esa época de enormes dificultades para acceder a materiales de construcción, con España tratando de reconstruir sus infraestructuras y Europa demandando acero para la Guerra Mundial, fue capaz de impulsar en 1943 la construcción del nuevo edificio detrás de la antigua Escuela de Ingenieros de Caminos. En él se acondicionó un espacio específico para una nueva Sección de Análisis Experimental de Estructuras ${ }^{8}$ (en el que se integró la unidad que ya existía de fotoelasticidad), dirigida por Carlos Benito 9 . De hecho, una de las mayores singularidades de la configuración del edificio, diseñado por el propio Torroja, radica en que las distintas zonas se encuentran estructuralmente aisladas para minimizar la transmisión de vibraciones entre zonas de ensayo.
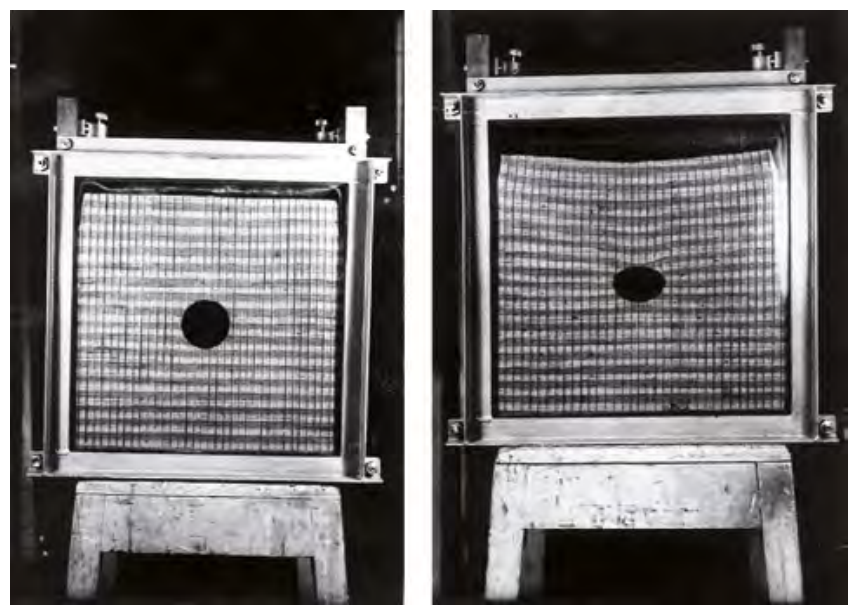

Figura 4. Modelo reducido del efecto de un túnel en un terreno arcilloso (1947).

8 El nuevo edificio se diseñó también para las Secciones de Mecánica del Suelo, de Ensayos Mecánicos, de Química y de Física.

9 Quien, tras fallecer Eduardo Torroja en 1961, tomó las riendas del Laboratorio; y que también sería posteriormente Director del CEDEX, y Catedrático y Director de la Escuela Técnica Superior de Ingenieros de Caminos, Canales y Puertos de Madrid. 


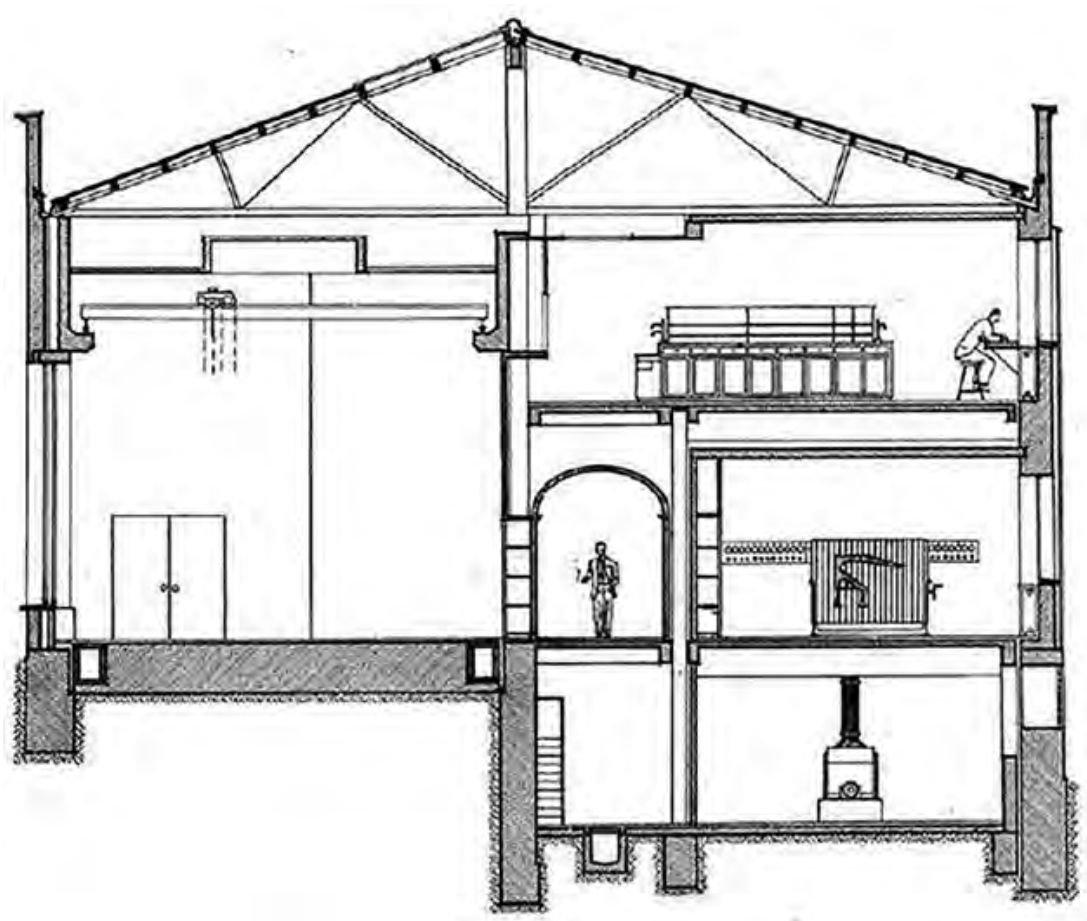

Figura 5. Sección del nuevo edificio del Laboratorio Central (1943). Se observa en una dependencia de la planta principal el modelo de ensayo de la cubierta del campo de Les Corts.

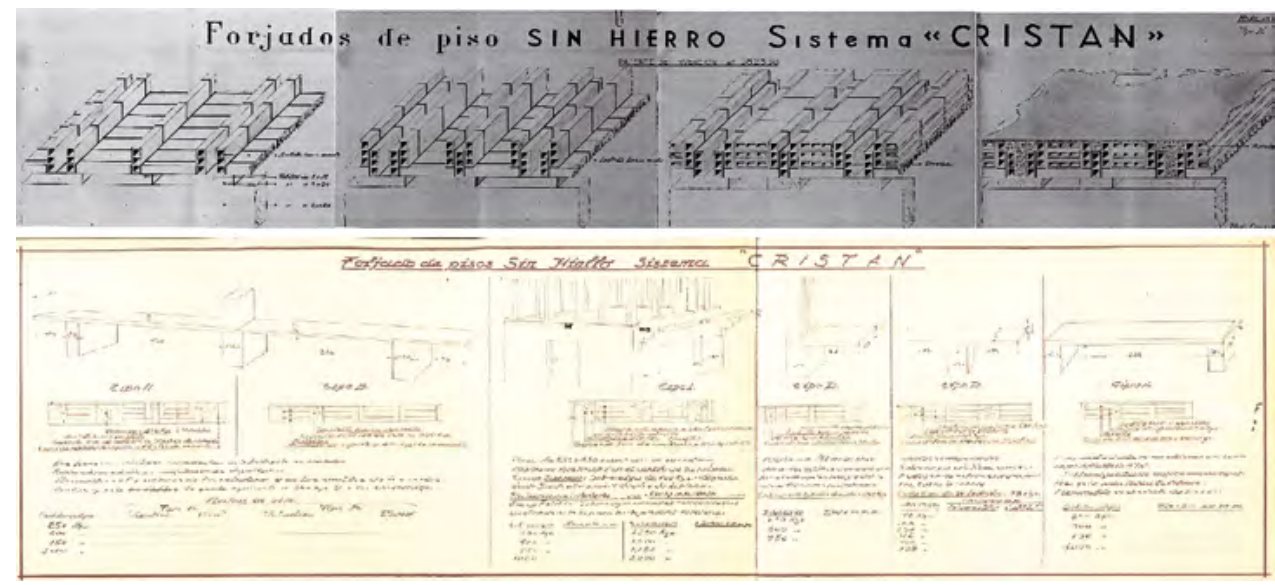

Figura 6. Patente y diseño de prueba de carga de un forjado de piso cerámico sin armadura (1944).

Los primeros años de reconstrucción del país tras la Guerra Civil generaron una fuerte recuperación de la actividad del Laboratorio. El cambio de paradigma introducido por Eduardo Torroja, y su propio prestigio personal, hicieron del Laboratorio una referencia para el ensayo de materiales y prototipos. Cabe mencionar la solicitud, en 1944, para el ensayo en el Laboratorio de una patente de un prototipo de forjado cerámico $\sin$ armadura mediante pruebas de $\operatorname{carga}^{10}$ (figura 6). Hay que tener en cuenta que la Segunda Guerra Mundial ocasionó una fuerte restricción de uso de los materiales de construcción. El cemento, y sobre todo el acero, quedaron bajo el control del Estado. De hecho, la Dirección General de Arquitectura publicó en esos años una serie de normas y reglamentos para forjados de edificación que buscaba (...) un mismo fin principal:

10 Forjado evidentemente no viable desde el punto de vista estructural. Los ensayos finalmente debieron hacerse por el Peticionario en la propia obra. Eduardo Torroja declinó elegantemente la invitación para presenciarlos. el ahorro del material metálico (...). Eso causó un problema de falta de calidad de las obras de construcción, en particular de las de edificación de promoción privada [2].

Los citados cambios de paradigma que trajo Torroja, organizativos y de instalaciones, supusieron el cimiento del crecimiento exponencial que el Laboratorio Central experimentó durante las siguientes décadas (figura 7), acompañando y apoyando al desarrollismo español de aquellos años.

4.

\section{LA ÉPOCA DE LOS MODELOS REDUCIDOS}

La década de los años 50 trajo dos nuevos campos de trabajo al Laboratorio. La realización de ensayos a petición de juzgados, lo que puede considerarse el germen de los estudios de 


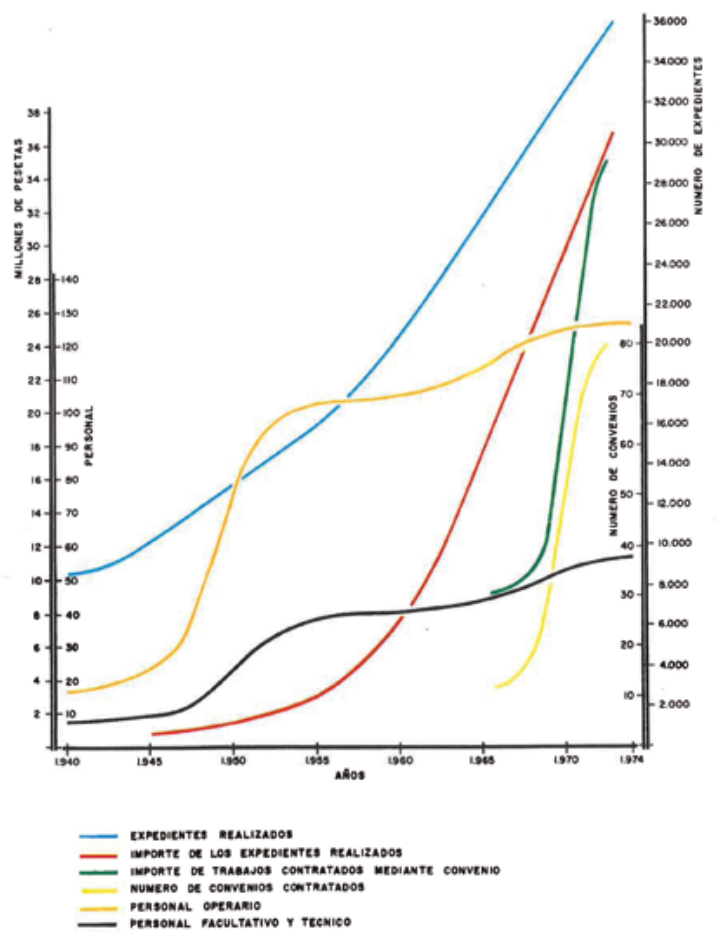

Figura 7. Evolución de la actividad del Laboratorio Central entre los años 40 y 70 del siglo XX.

patología estructural; y los ensayos sobre modelos reducidos de presas (figura 8), que se estuvieron realizando hasta la década de los años 80. En el Laboratorio Central se ensayaron buena parte de las grandes presas construidas en aquellos años al amparo de las políticas nacionales de desarrollo (Aldeadávila, Almendra, Riaño, Atazar, etc).

Los ensayos sobre modelos reducidos de presas comenzaron a realizarse en Portugal e Italia, de donde llegó la tecnología al Laboratorio Central a mediados de los años 50. No obstante, ya a finales de los años 40 , dentro de las investigaciones propias desarrolladas en el Laboratorio, se habían realizado ensayos sobre modelos fotoelásticos de presas de contrafuertes fabricadas con materiales de muy bajo módulo elástico y alta densidad, como el "Litarmul"; y se había ensayado el empleo de gelatinas para la fabricación de los modelos.

Fruto de estas investigaciones, los primeros modelos en los años 50 se construían con "Litargel" (producto desarrollado en el propio Laboratorio mezcla de gelatina, glicerina, litargirio y agua a $50^{\circ} \mathrm{C}$ ). Este producto tenía un bajo módulo de deformación, lo que permitía generar el empuje sobre la presa con aceite de oliva.

Entre los primeros modelos reducidos de presas cabe destacar los realizados para investigar el colapso de la presa de Vega de Tera, en Zamora, cuya rotura el 9 de enero de 1959 provocó la catástrofe de Ribadelago ${ }^{11}$. Se realizaron varios modelos entre 1959 y 1960 que permitieron establecer las causas de su hundimiento. La presa había sido construida entre 1954 y 1956. Para hacer frente a la escasez de cemento se planteó la construcción de una presa de pantalla de hormigón con contrafuertes de mampostería. El informe sobre su colapso lo firmó el propio Eduardo Torroja poco antes de su fallecimiento. En dicho informe se apuntó que la presa de Vega de Tera se hundió por defecto de concepción y la causa fundamental del hundimiento fue la diferencia de los módulos de elasticidad del hormigón y de la mampostería. En efecto, en el proyecto de la presa se asumió un módulo elástico de $9.810 \mathrm{~N} / \mathrm{mm}^{2}\left(100.000 \mathrm{kp} / \mathrm{cm}^{2}\right)$ para la mampostería, mientras que los ensayos que se hicieron para el estudio de las causas de su hundimiento reflejaron un módulo de solo $981 \mathrm{~N} / \mathrm{mm}^{2}$ (10.000 kp/ $\left.\mathrm{cm}^{2}\right)$. De este modo, en la práctica la contribución de los contrafuertes fue notablemente menor de la concebida, lo que provocó la rotura de la pantalla de hormigón. Este fue uno de los primeros estudios de patología estructural realizados al amparo del Laboratorio.

Posteriormente, se pasó a fabricar los modelos reducidos de presas con mezclas plásticas, que tenían un módulo de elasti-

11 El colapso de la presa afectó al vecino pueblo de Ribadelago, donde fallecieron 144 personas. Fue el segundo colapso de una presa por número de fallecidos, tras la presa de Puentes II en 1802, cuyo informe de causas redactó Agustín de Betancourt y motivó la creación del Cuerpo de Ingenieros de Caminos, Canales y Puertos.
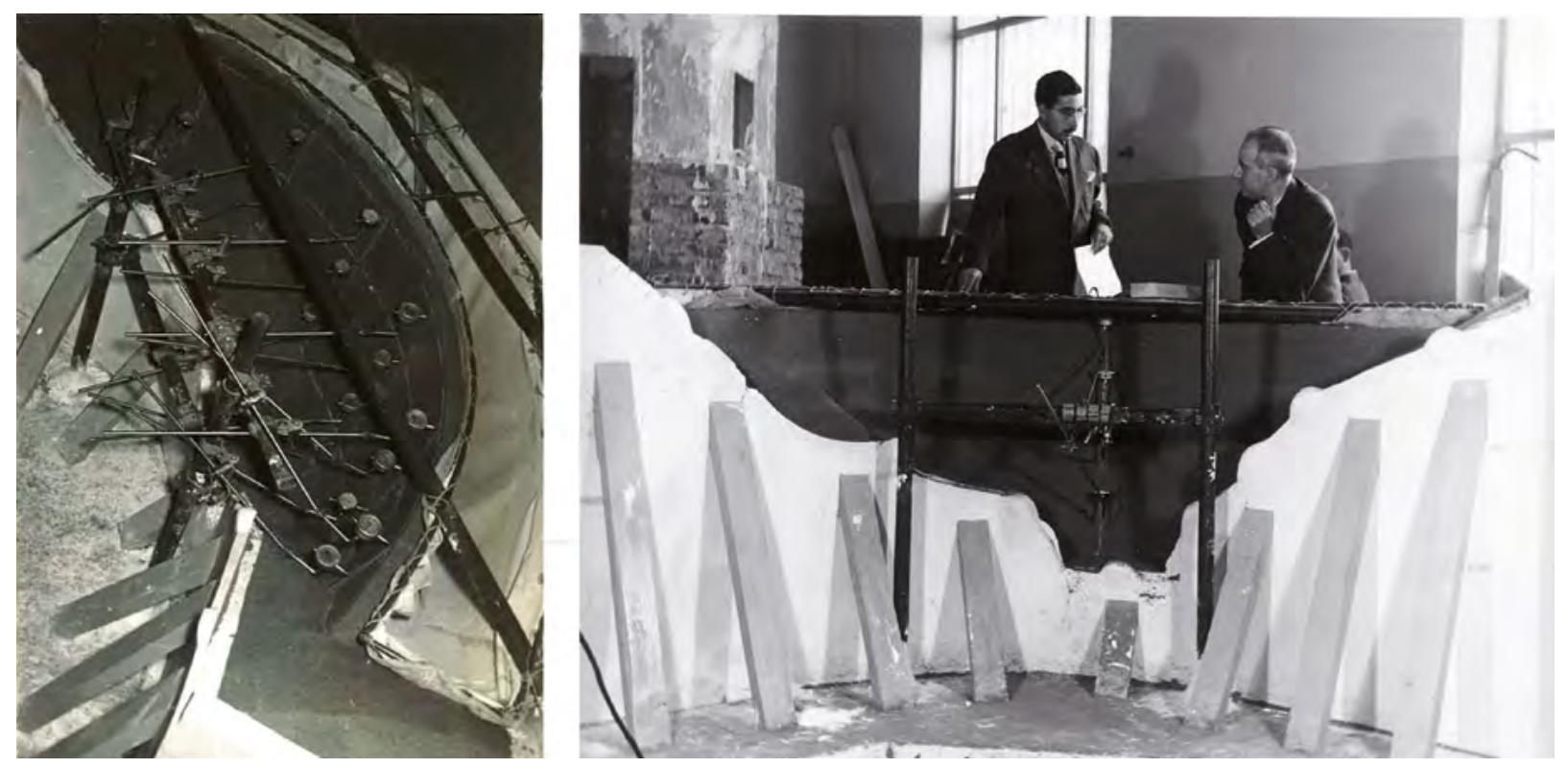

Figura 8. Modelo reducido de la presa de Santa Ana. 

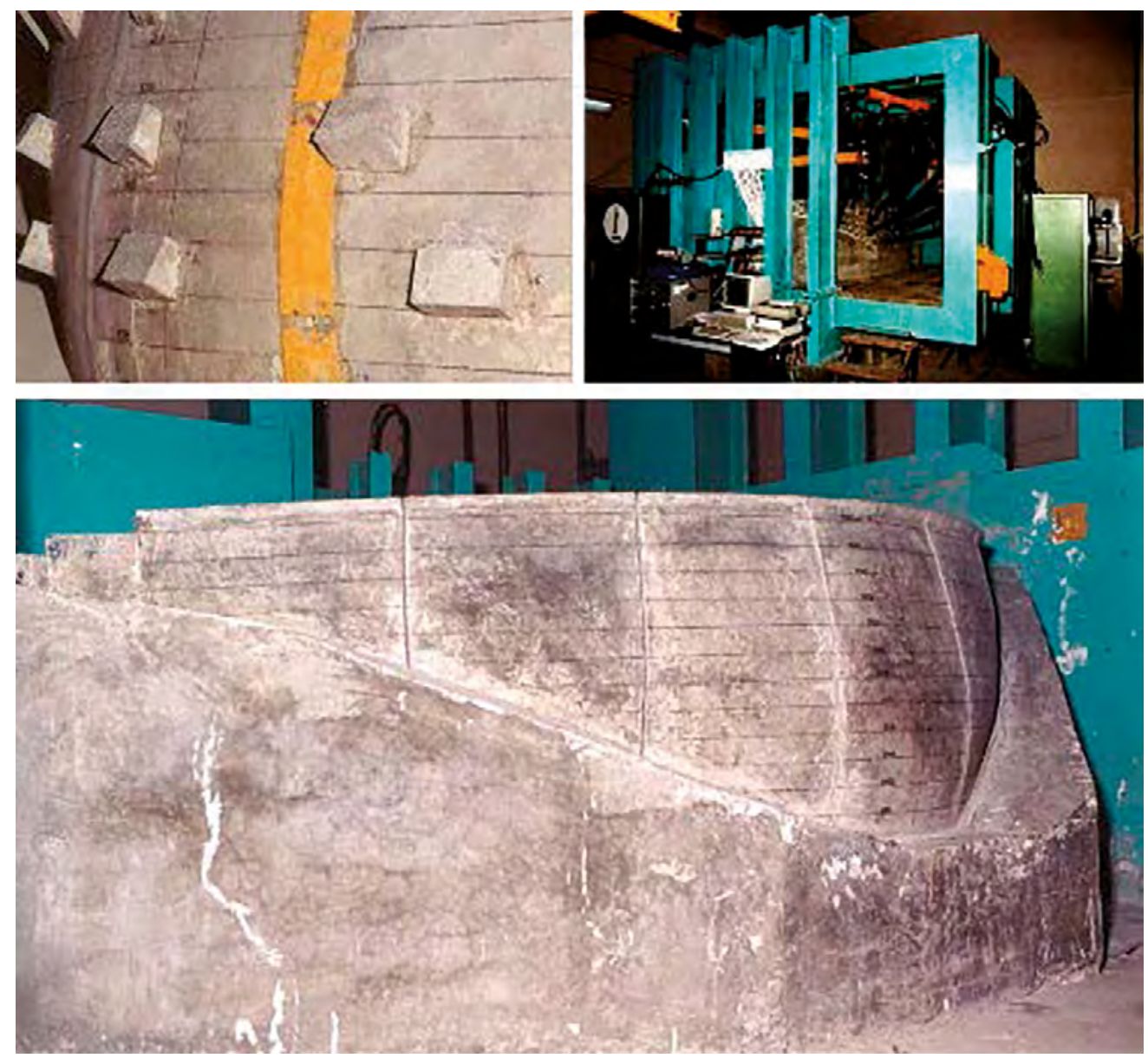

Figura 9. Modelo reducido de la presa de Las Cogotas.

cidad superior, por lo que los empujes se inducían con mercurio. Y finalmente, desde los años 60 , los modelos de presas se construían con microhormigones sobre moldes de encofrado de escayola (figura 9). El empuje se materializaba con actuadores hidráulicos. Los actuadores podían movilizar los empujes horizontales, mientras que las acciones verticales se reproducían mediante tirantes embebidos a distintas alturas en la masa del modelo; o, más adelante, se encofraban dados en el trasdós de las presas para el apoyo de los actuadores que movilizaban conjuntamente el empuje vertical y horizontal.

Además de los modelos de fotoelasticidad y los de presas, desde finales de los años 40 y hasta mediados de los años 70 , se realizaron numerosos ensayos sobre modelos reducidos de otros tipos de estructuras, como losas (esviadas, reticulares y ortótropas), puentes, bóvedas y, sobre todo, cubiertas laminares $^{12}$. Estos ensayos inicialmente se realizaban dentro de trabajos de investigación interna del Laboratorio, pero pronto sirvieron para atender peticiones de algunos de los más insignes Ingenieros de la época: el mismo Torroja, Ildefonso Sánchez del Río, Carlos Fernández Casado, Arendt M. Haas, etc. Además, los talleres del Laboratorio Central suministraban numerosos modelos a otros organismos de investigación, como el Instituto Técnico de la Construcción y del Cemento.

12 Eduardo Torroja fue uno de los fundadores de la IASS (International Association for Shell and Spatial Structures), cuya Secretaría lleva establecida desde entonces en el Laboratorio Central.
Algunos de los modelos más representativos ensayados en esta época fueron el del puente sobre el río Caudal en Mieres (1966); o las cubiertas del Club Tachira en Caracas (1957, anteproyecto), del edificio Bacardí en La Habana (figura 10), del canódromo de Madrid (1961), del Palacio de Congresos de Barcelona (1961), de la iglesia de Nuestra Señora de Guadalupe en Madrid (1963) y del Palau Blaugrana en Barcelona (1971).

Los primeros modelos de estructuras eran de tipo elástico, realizados en plexiglás ${ }^{13}$ (figura 11). Posteriormente, estos modelos se combinaban con otros realizados con microhormigones armados con alambres, e incluso postesados, para modelizar los modos de rotura (figura 12). Hay que hacer notar que es en esta época cuando, con la aparición de la Instrucción HA-61 [3] por el impulso del propio Eduardo Torroja y Alfredo Páez, se abandona el cálculo elástico determinista de tensiones admisibles en secciones para pasar al cálculo semiprobabilista plástico en agotamiento.

También se ensayaron otros modelos de elementos estructurales singulares, entre los que caben destacar las tomas del salto de Valdecañas, una vasija de reactor nuclear, una torre de distribución eléctrica, andamios, etc. Durante toda esta época el responsable de estos trabajos, bajo la dirección de Torroja, fue el citado Carlos Benito.

En todos estos ensayos sobre modelos reducidos las medidas de las deformaciones se tomaban con comparadores y

13 Polimetacrilato de metilo (PMMA). 

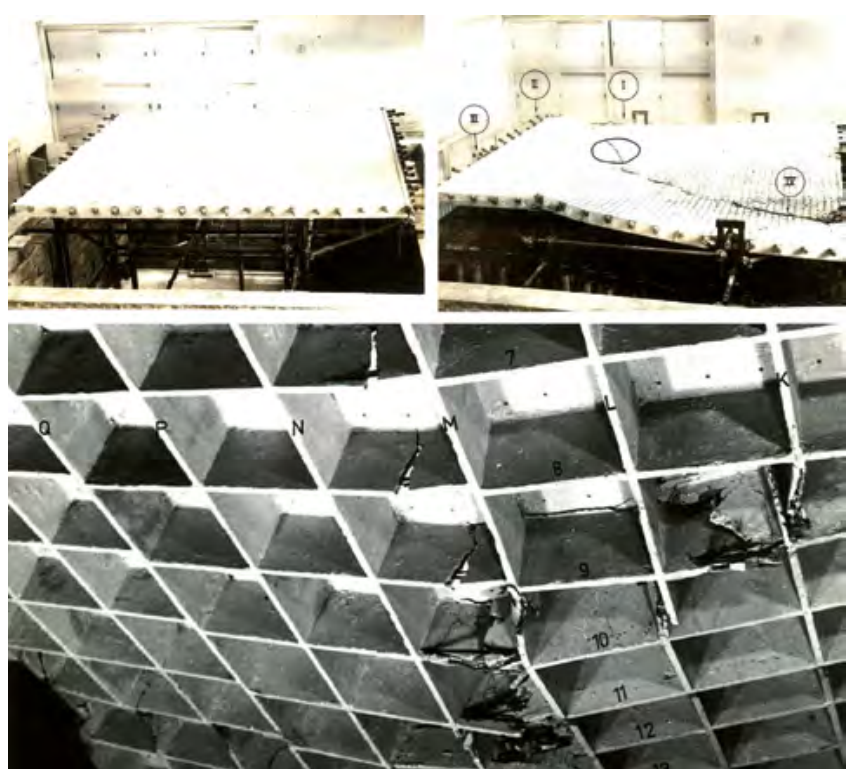

Figura 10. Modelo reducido de un forjado en el edificio Bacardí en La Habana (1960).

bandas extensométricas ${ }^{14}$. La cada vez mayor rigidez de los modelos exigió que su auscultación se realizase con sensores de mayor precisión, pasando de extensómetros mecánicos a eléctricos. El propio Laboratorio desarrolló en estos años algunos prototipos de sensores para medición de deformaciones.

Como se ha dicho, también desde principios de la década de los 50 el Laboratorio comenzó a colaborar en asuntos judiciales elaborando informes, primero sobre características de materiales de construcción, y luego ya de carácter pericial (como, por ejemplo, el estudio en 1967 de las causas del hundimiento de la presa

14 Uno de los primeros fue el modelo GM4476 de Philips.
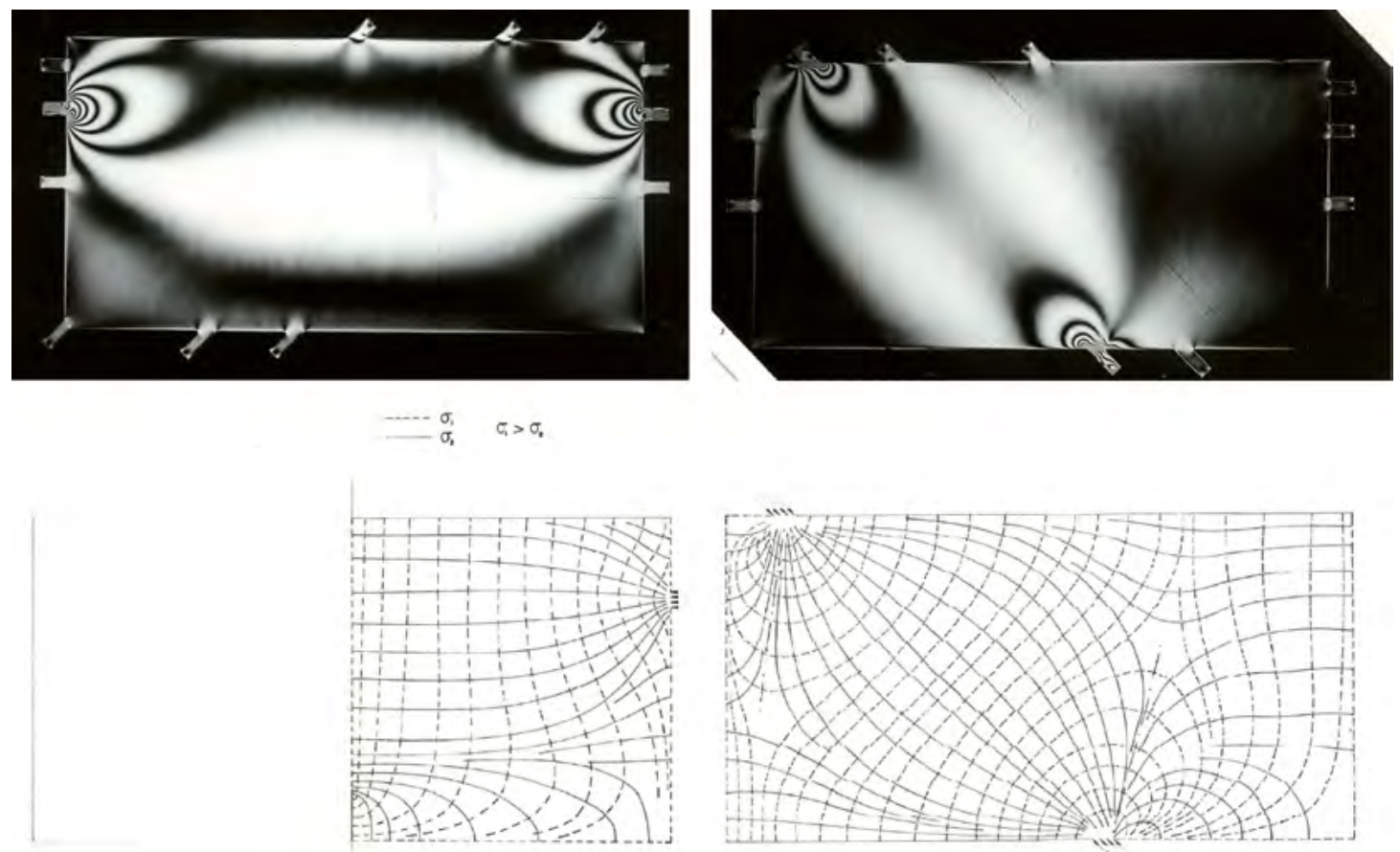

Figura 11. Modelo fotoelástico para análisis de la transferencia de pretensado (1969). 

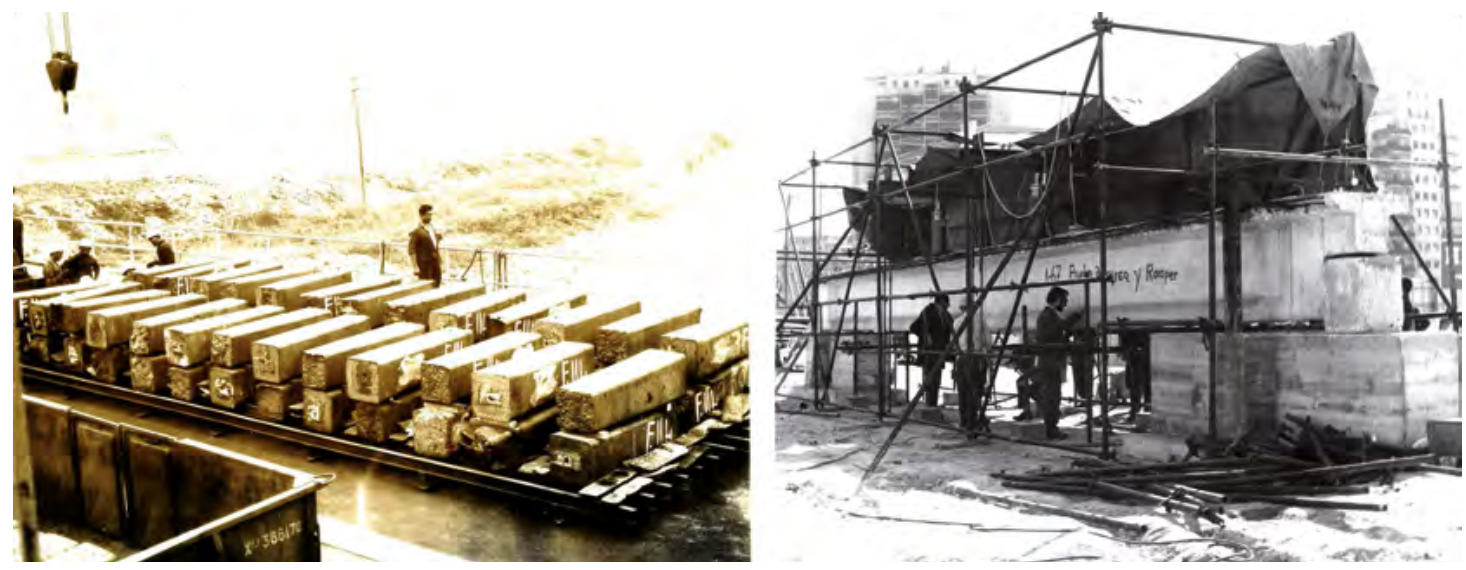

Figura 12. Pruebas de carga en un puente del embalse de las Segadas (1964) y de una viga en AZCA (1969).
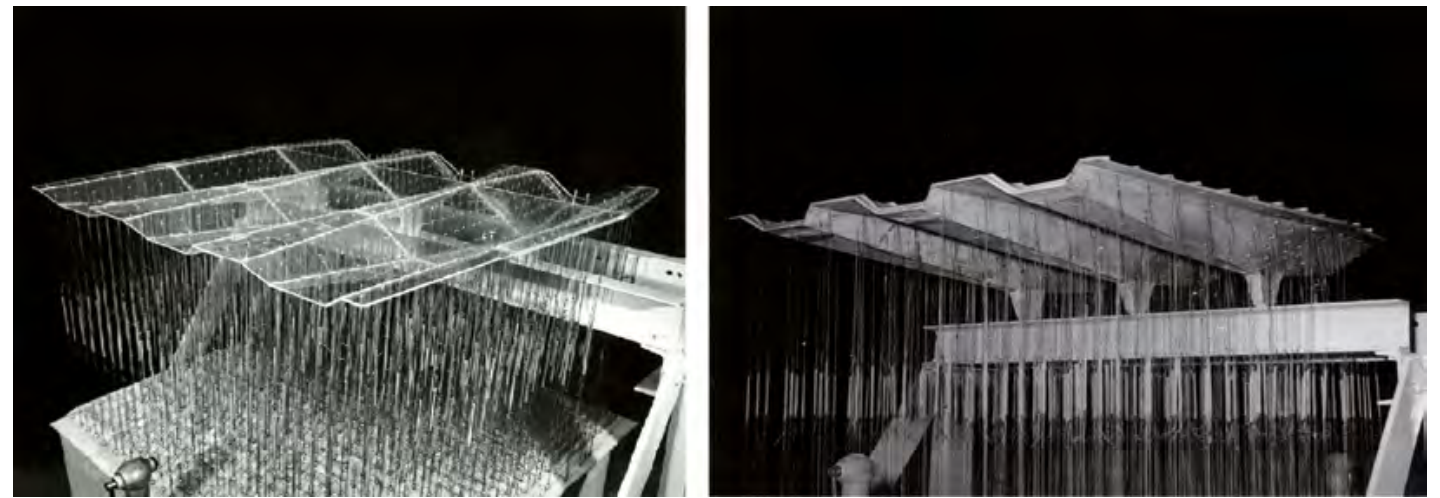

Figura 13. Modelos reducidos (elástico y de rotura) de la cubierta del Canódromo de Madrid (1961).

nando de Arriaga; fotoelasticidad, con Juan Moreno Torres; y se creó un nuevo equipo para la realización de pruebas de carga estáticas y dinámicas bajo la coordinación de Juan Granell Vicent (quien se convertiría en subdirector del Laboratorio).

Las primeras pruebas de carga se realizaron sobre puentes de carretera (entre otros, los de la carretera nacional N-630 sobre el Almonte y el Tajo en la Vía de la Plata), aunque pronto se extendieron a los puentes de ferrocarril, realizándose en pocos años más de sesenta pruebas (figura 13).

Durante los primeros años de la década de los 70, cabe destacar las pruebas de carga realizadas en varios pasos superiores de Madrid (Paseo de la Castellana, Raimundo Fernández Villaverde, Santa María de la Cabeza, etc) y en numerosos forjados de edificación del complejo comercial AZCA (figura 13).

La construcción de muchas estructuras fue apoyada desde su diseño hasta su puesta servicio por ensayos en el Laboratorio Central. Como ejemplo se puede mencionar el viaducto sobre el río Cigüela en el Trasvase Tajo-Segura: en el Laboratorio se llevaron a cabo ensayos fotoelásticos y sobre un modelo reducido elástico para analizar las tensiones de transferencia del pretensado de los vanos; y con la obra ya construida se realizaron las pruebas de carga (figura 14).

Por supuesto se continuó con la realización de ensayos mecánicos sobre nuevos materiales, como fueron las barras de armado de alto límite elástico de dureza natural de REA (1968), NERSID (1965) o ALTRES. Asimismo, cabe destacar la realización de ensayos de fuego sobre materiales refractarios para la protección de elementos metálicos, o el empleo de rayos $\mathrm{X}$ con un equipo portátil para la detección de armaduras en tubos.

También durante los años 70 se iniciaron los ensayos de relajación, fractura, fatiga y corrosión bajo tensión en alambres de pretensado. En este campo trabajaron en el Laboratorio Manuel Elices Calafat ${ }^{16}$, Vicente Sánchez Gálvez, y José Climent, hasta poner a punto el ensayo acelerado de tiocianato amónico que actualmente se utiliza para evaluar la susceptibilidad de estos aceros a la corrosión bajo tensión.

\section{6.}

\section{LUIS ORTEGA BASAGOITI Y LAS INSPECCIONES SISTEMÁTICAS DE ESTRUCTURAS}

El citado Juan Moreno Torres (entre 1971 y 1973) y José María Morera Bosch (entre 1973 y 1976) precedieron a Luis Ortega Basagoiti como responsables del Servicio de Estructuras. Con la jefatura de Luis se reorganizó y amplió significativamente esta unidad de estructuras, aglutinando distintos tipos de trabajos: el análisis experimental de estructuras (modelos) con Rafael Astudillo y Javier Sainz de Cueto; la auscultación de obras construidas con Jesús Galindo; las inspecciones sistemáticas de puentes de RENFE con Mariano Garzo y Juan Luis

16 Quien ya en 1966 estaba trabajando en estudios especiales de materiales. 

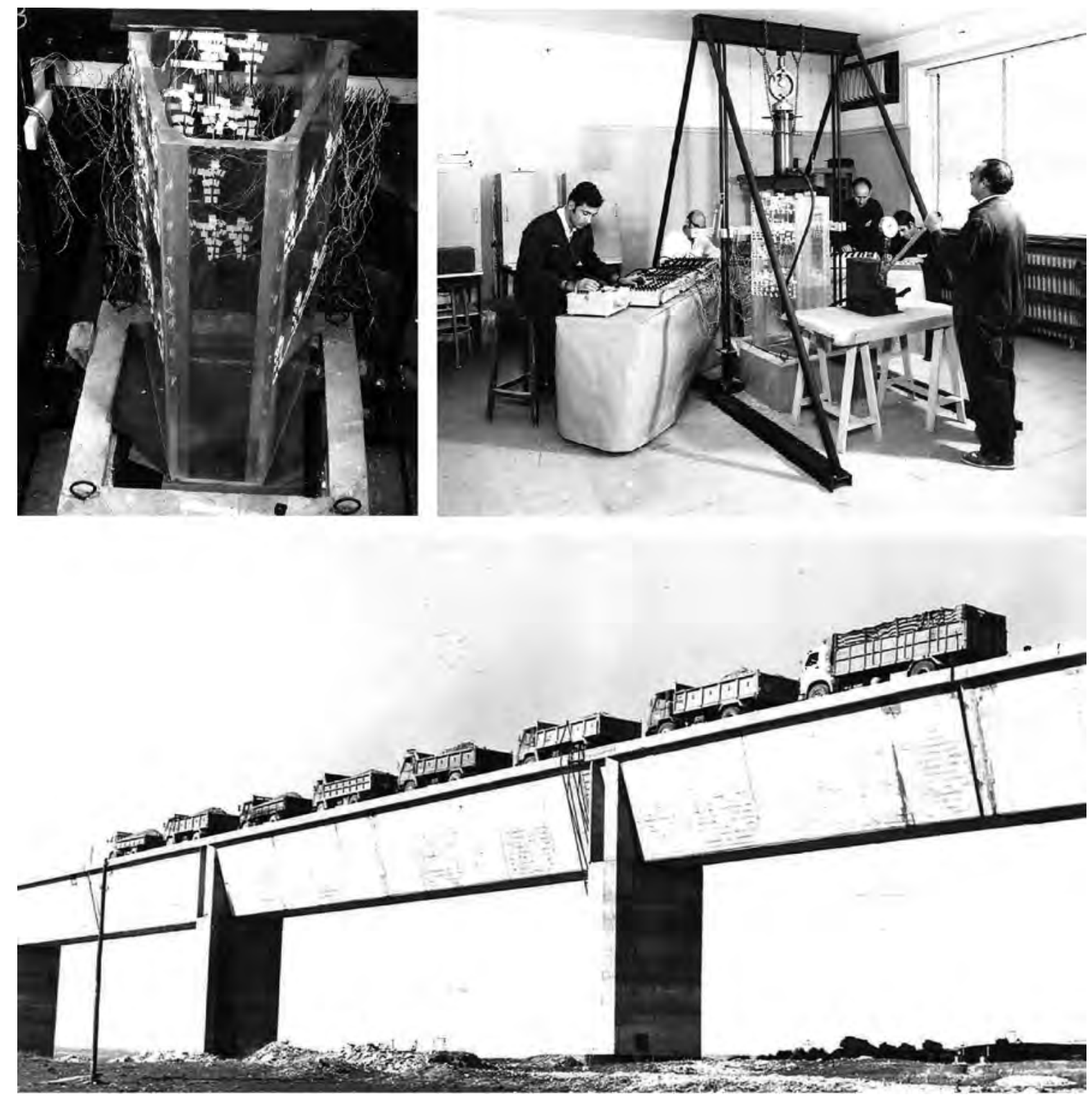

Figura 14. Ensayos en modelo reducido y pruebas de carga en un acueducto del trasvase Tajo-Segura (1969-1970).
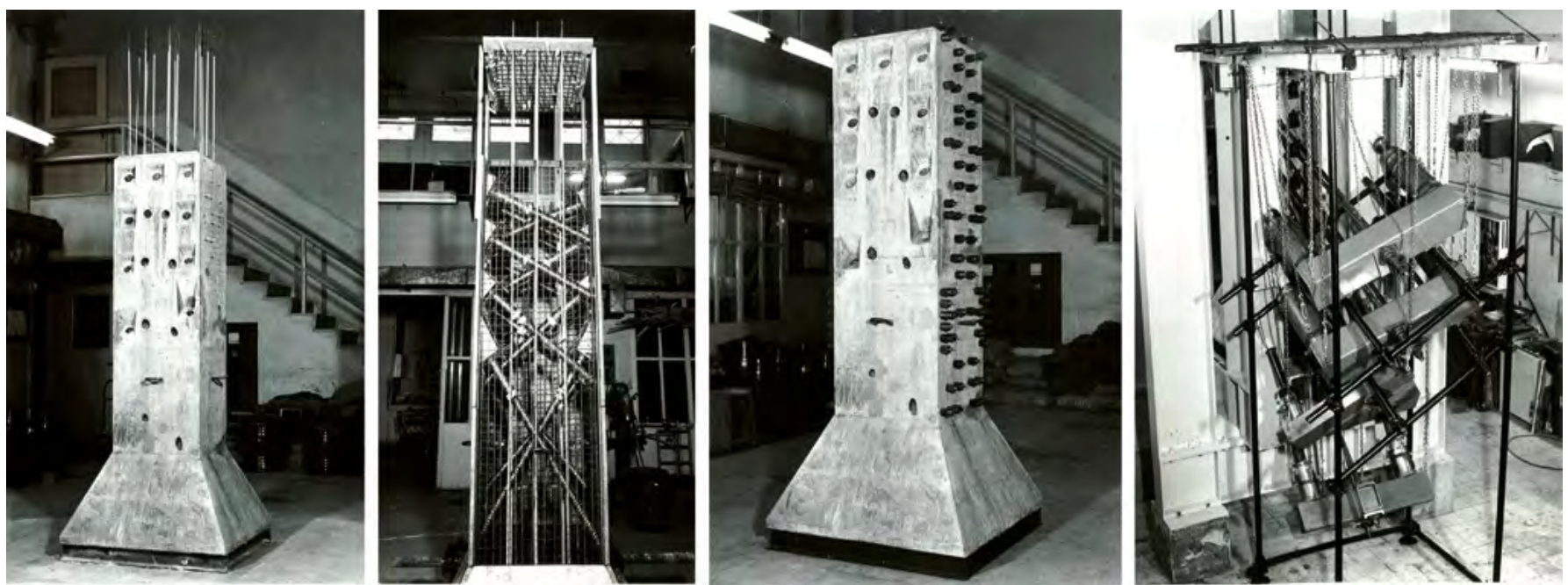

Figura 15. Ensayos en modelo reducido de la cabeza de pila del puente de Rande (1975-1977). 

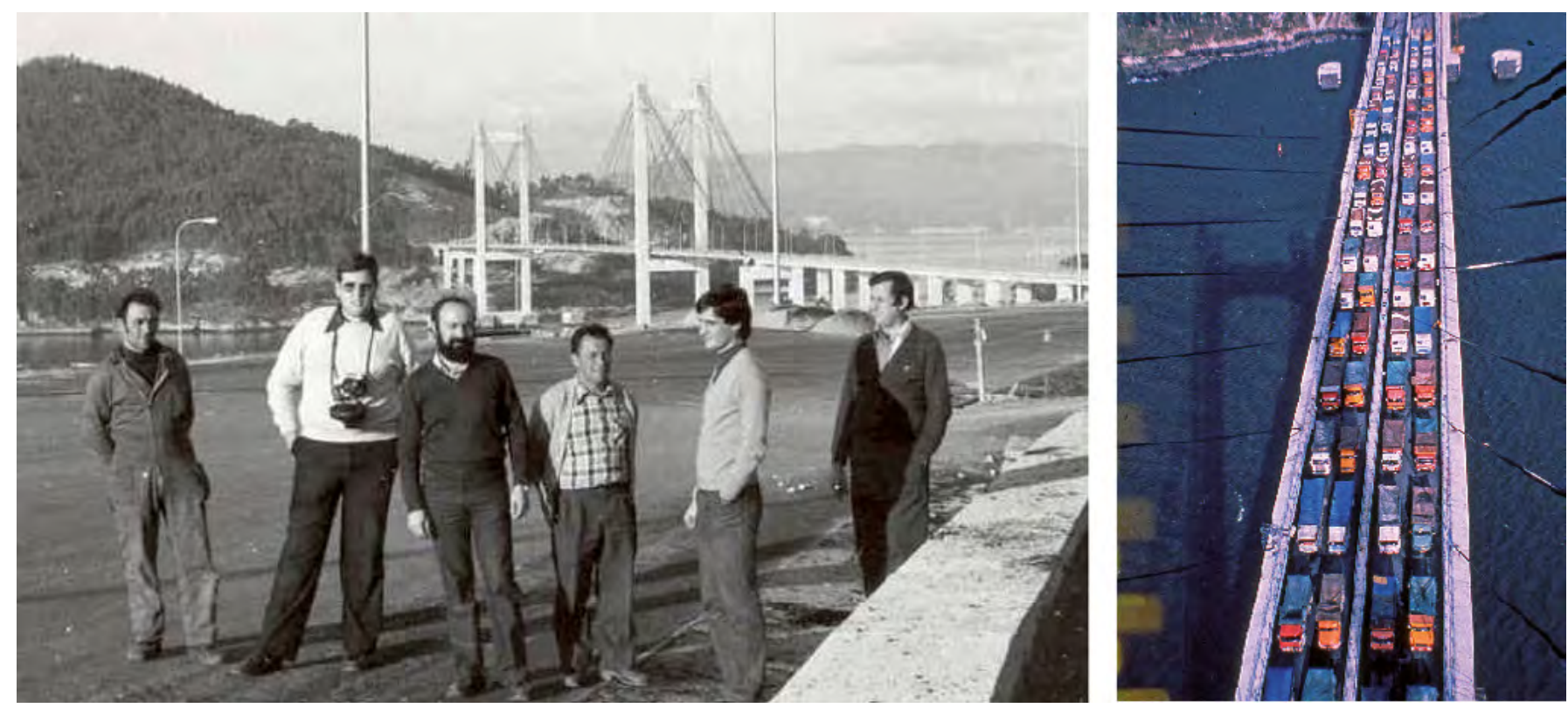

Figura 16. Prueba de carga del puente de Rande (1975-1977). Personal del Laboratorio Central: Luis Ortega y Rafael Astudillo (segundo y tercero por la izquierda) y Heliodoro García y Carlos Castejón (primero y segundo por la derecha).

Alcalá; y los estudios teóricos con Teófilo Serrano ${ }^{17}$. Es en esta época cuando arrancó como línea de trabajo fundamental la inspección y auscultación de estructuras, línea que continúa vigente hoy en día.

Entre los modelos ensayados en esta última época destaca un sistema de remolque de vagones Talgo para RENFE. Este trabajo tendría continuidad con el ensayo de un cambio de vía que sería el germen del actual cajón ferroviario del CEDEX. También se ensayó un modelo reducido de la cabeza de una pila del puente de Rande para analizar las tensiones inducidas por el desvío de los cables (figura 15).

También en estos años se realizaron numerosas pruebas de carga en forjados y puentes (puente de La Salve, viaducto del embalse de García Sola, aparcamiento subterráneo de la calle Almagro, puente sobre el Tormes en Salamanca, puente de Triana, urbanización Molino de la Hoz, etc). De entre todas ellas cabe destacar las realizadas para la puesta en servicio del mencionado puente de Rande encabezadas por Luis Ortega (figura 16).

Otra de las iniciativas de aquellos años fue la construcción de la primera pasarela de inspección de puentes de carretera con diseño de Jesús Galindo. Se trataba de una estructura metálica modular que se montaba en campo y se transportaba y manipulaba mediante un camión dotado con una pluma. Esta pasarela se utilizó, entre otros, en los puentes de Almodóvar del Río y del estrecho sobre el río Sil.

Durante esta época el Laboratorio comenzó a desarrollar una incipiente actividad en el campo de la patología en la edificación, hasta entonces solo atendida por ensayos sobre materiales o productos, o por la realización de pruebas de carga en forjados. Así se estudiaron los efectos de un incendio en el

17 En colaboración con el Centro de Cálculo que se había creado en el Ministerio de Fomento, en el que estaba, entre otros, Avelino Samartín Quiroga, que había trabajado antes en el Laboratorio Central. complejo AZCA o los daños aparecidos en la estación de tren de San Bernardo en Sevilla a raíz de la construcción del Metro.

En el ámbito de los estudios teóricos se estableció una estrecha colaboración con la Dirección General de Carreteras para el desarrollo de normativa sobre acciones y sobre el empleo de aceros de pretensado.

\section{7.}

\section{LA ÉPOCA DE LOS CAMBIOS}

A mediados de los años 80 se produjeron una serie de cambios organizativos en el CEDEX que se reflejaron en el Laboratorio Central. Entre otros aspectos, dada la importante actividad del Laboratorio en el ámbito de las estructuras, se cambió el nombre del mismo al actual ${ }^{18}$. En 1987 se hizo cargo del Sector de Auscultación de Estructuras Rafael Astudillo, y del Sector de Estudios Juan Luis Alcalá. Este Sector de Estudios se encargó del análisis del hundimiento del polideportivo de Huesca (1993).

Bajo la coordinación de Mariano Garzo, y con la incorporación de Jorge García Reig al equipo, durante estos años se continuó realizando las inspecciones para RENFE, estudiándose hasta la década de los años 90 más de doscientos puentes. Estas inspecciones no solo se centraban en la patología que pudieran presentar, sino también en la descripción de su tipología y de su geometría; y permitieron disponer de una base de datos para su gestión. En el caso de los puentes más singulares las inspecciones se complementaban con pruebas de carga, como en Tuy, Alsasua (La Salera), Herrera del Duque (Guadámez y Guadalefra), etc.

18 De Laboratorio Central de Ensayo de Materiales de Construcción pasó a llamarse Laboratorio Central de Estructuras y Materiales. 

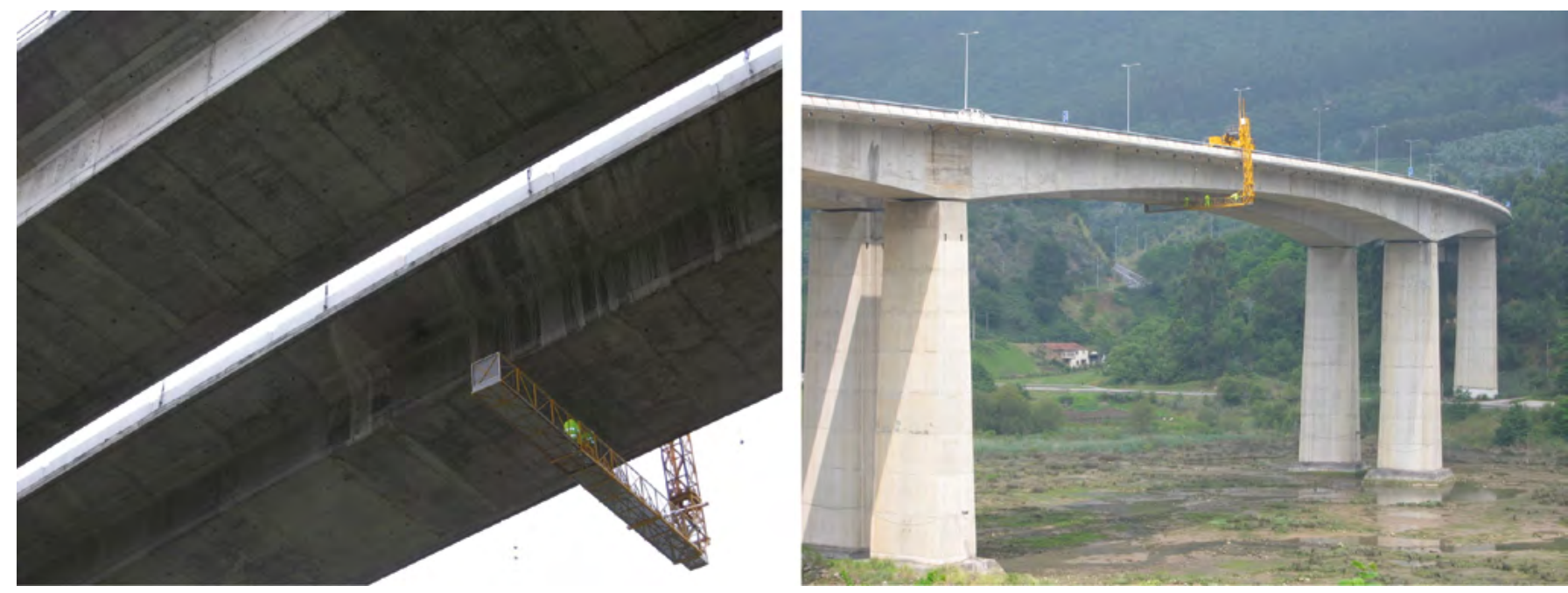

Figura 17. Inspección de los puentes de Ontón y Oriñón (1998).

A principios de los años 80 se abrieron nuevas perspectivas en el campo de las estructuras. Pablo Quintanilla se hizo cargo de una nueva unidad de ensayos dinámicos, que sería el germen del área actual que gestiona la mesa sísmica del Laboratorio. También se creó una unidad especializada en estudios de edificación, dirigida por Antonio de las Casas, y en la que trabajaban Teresa González Limón y Mónica Álvarez de Buergo. Esta unidad realizó durante los años 90 numerosos estudios sobre edificios históricos, entre los que cabe destacar las torres de la Catedral de San Martín en Teruel, el Palacio de los Botines de León, y la Torre Comares de la Alhambra.

A caballo entre los años 80 y 90 se siguieron haciendo pruebas de carga estáticas (cada vez menos) y dinámicas (cada vez más), como en el viaducto del Pretorio; en los de Carballiño y Verín; en el puente del Kursaal sobre el Urumea; en los viaductos de Tordesillas, Benavente, Alcanadre; y en varios puentes construidos con motivo de la Exposición Universal de Sevilla en 1992, entre los que destaca el puente del Centenario sobre el Guadalquivir. En total en esta época se realizaron más de sesenta ensayos de este tipo.

En el campo de la innovación en materiales de construcción se llevaron a cabo investigaciones sobre los hormigones de alta resistencia y la durabilidad de las estructuras de hormigón, con Pilar Alaejos y Fernando Rodríguez respectivamente, ambos coordinados por José Manuel Gálligo; investigaciones cuyos resultados se incorporaron a la Instrucción EH-98 [4].

\section{8.}

\section{EL CAMIÓN PASARELA DE INSPECCIÓN DE PUENTES}

En 1995 se hizo cargo del Sector de Auscultación de Estructuras el mencionado Mariano Garzo. Bienvenido Martínez Fernández, quien hasta entonces había formado parte del equipo de Mariano Garzo, se hizo cargo del Sector de Estudios de Estructuras, incorporando la unidad de estudios de construcciones históricas que hasta entonces había dirigido Antonio de las Casas. Este equipo realizó numerosos estudios por encargo del Ministerio de Educación y Ciencia. Ya iniciado el siglo XXI se realizó, con apoyo del Sector de Auscultación, una serie de trabajos de asistencia técnica para la rehabilitación del Casón del Buen Retiro; y se inició un programa de auscultación de presas (SIGAP).

Durante estos años colaboraron en ambos sectores distintos ingenieros, como Francisco Navarro, quien posteriormente se haría cargo de un nuevo Sector de Dinámica de Estructuras que se encarga desde entonces de la gestión de la mesa sísmica del Laboratorio.

Con Mariano Garzo al frente, y dentro de sucesivos convenios con la Dirección General de Carreteras del Estado, se realizaron más de ciento ochenta inspecciones de puentes, muchas de ellas para el estudio de la fluencia de puentes construidos por avance en voladizo (viaductos de Ontón y Oriñón en la A8, viaducto del Estrecho sobre el río Sil, etc). Para ello se sustituyó la antigua pasarela de inspección por una nueva desplegable montada sobre un camión (figura 17), lo que agilizó significativamente los trabajos y permitió incrementar el número de puentes inspeccionados. La pasarela se inauguró en el viaducto del Azufre sobre el río Sil, en Ponferrada. Un convenio similar se suscribió con la Confederación Hidrográfica del Guadiana durante la segunda mitad de los años 90, inspeccionándose buena parte de los puentes de su red viaria.

El equipo de estudios avanzados de estructuras, bajo la coordinación de José María Arrieta, colaboró en la implantación de los Eurocódigos llevando a cabo su traducción y el desarrollo del Documento Nacional de Aplicación del Eurocódigo 2 [5]. Este equipo realizaba estudios de evaluación estructural, entre los que cabe destacar en 1998 el estudio de las causas de la rotura de un depósito en Melilla y del colapso de la cubierta del Pabellón de Ermua.

\section{9.}

\section{LA LLEGADA DEL SIGLO XXI}

Durante los primeros años del siglo XXI se produjo una fuerte descapitalización de personal adscrito al CEDEX, por lo que en 2005, con Pablo Díaz Simal al frente, se unificaron todas las 

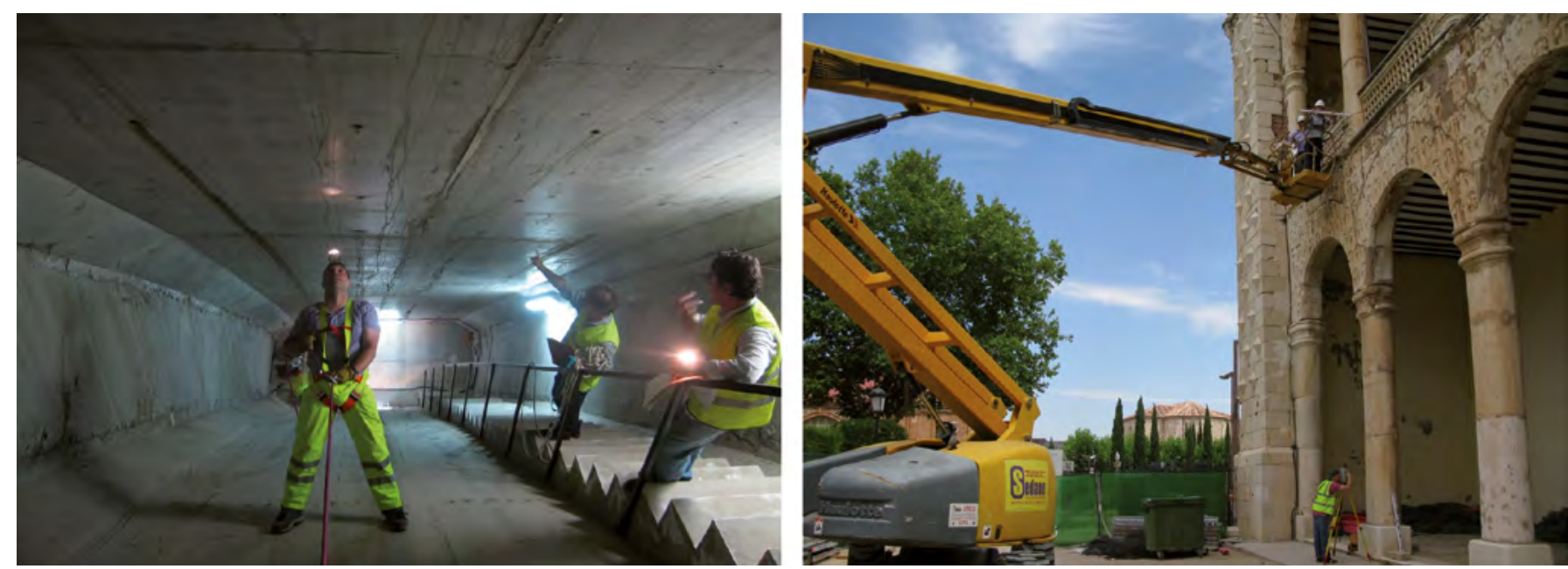

Figura 18. Inspección del puente sobre el río Almonte de la A-66 (2009) y del Palacio del Infantado de Guadalajara (2018).
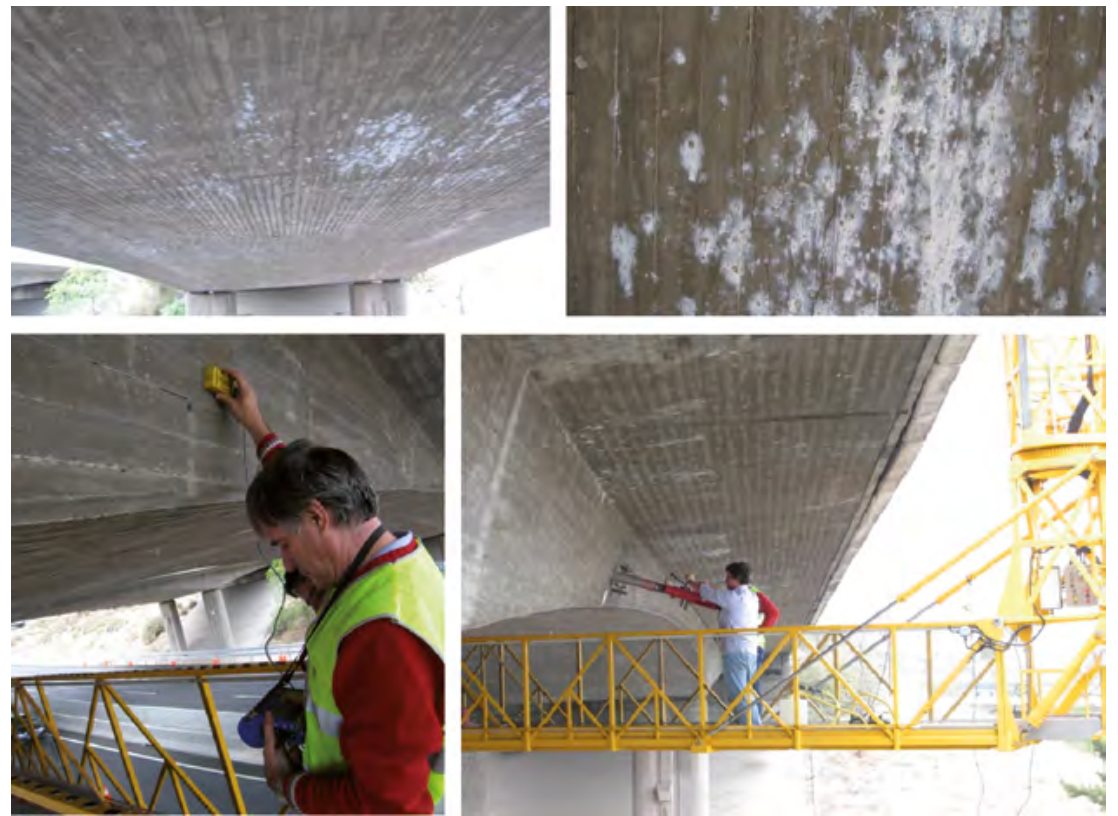

Figura 19. Estudio de un puente de hormigón postesado afectado por un ataque árido-álcali (2009).

unidades dedicadas al ámbito de las estructuras en una única Área de Estudios y Auscultación de Estructuras. En estos años se mantuvo la estrecha colaboración con la Dirección General de Carreteras para el estudio de puentes con problemáticas singulares (del orden de ciento cincuenta puentes fueron analizados entre 2003 y 2018), como los puentes de la A63 sobre el Tajo y el Almonte (figura 18), el puente de Amposta de Ribera, el viaducto de Contreras, el viaducto sobre el embalse de Rules, el puente de Alcoy, etc. Muchos de estos estudios se complementaron con ensayos dinámicos ${ }^{19}$. Para ADIF cabe destacar el estudio realizado sobre las anomalías existentes en el viaducto sobre el río Arlanzón de la LAV Madrid-Burgos.

En 2007, por encargo de ADIF, se puso en marcha por primera vez un sistema de adquisición remota en tiempo real de

19 En aras de analizar la evolución del comportamiento estructural de los puentes, gracias a que la amplia experiencia del Laboratorio en este tipo de ensayos permite disponer de resultados de ensayos anteriores realizados sobre las mismas estructuras. datos de auscultación de dos grandes viaductos en Sant Boi, en la entrada del AVE a Barcelona desde Zaragoza. Esta técnica se depuraría en la instrumentación del puente del Candí, en la misma línea, para evaluar la eficacia de la reparación mediante el control en tiempo real de los movimientos del puente. Posteriormente se ha utilizado en otras ocasiones, como en el citado puente de Amposta.

En 2009 se realizó un estudio sobre sendas estructuras de la autopista A42 sobre la N400 en Toledo, identificándose y evaluándose un proceso de degradación del hormigón en las losas postesadas de los tableros por reacciones tipo árido-álcali ${ }^{20}$ (figura 19). Desde entonces se han identificado varias estructuras más en España ${ }^{21}$ con procesos patológicos similares, sobre las que se han realizado estudios de evaluación estructural y

20 Fenómeno ya estudiado en el Laboratorio Central desde los años 80 por Jesús Soriano en el campo de las presas.

21 Se tiene referencia también de problemas similares en otros países detectados en estos últimos años. 

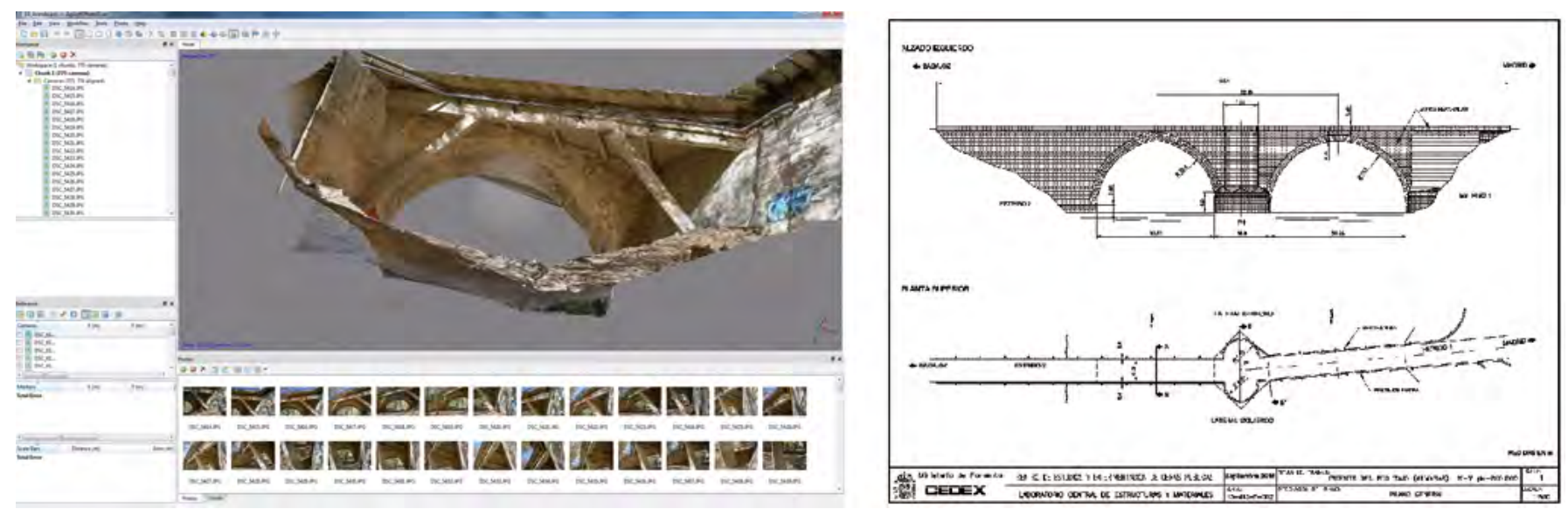

Figura 20. Estudios de caracterización de puentes históricos con cargo al 1\% Cultural (2014-2017).
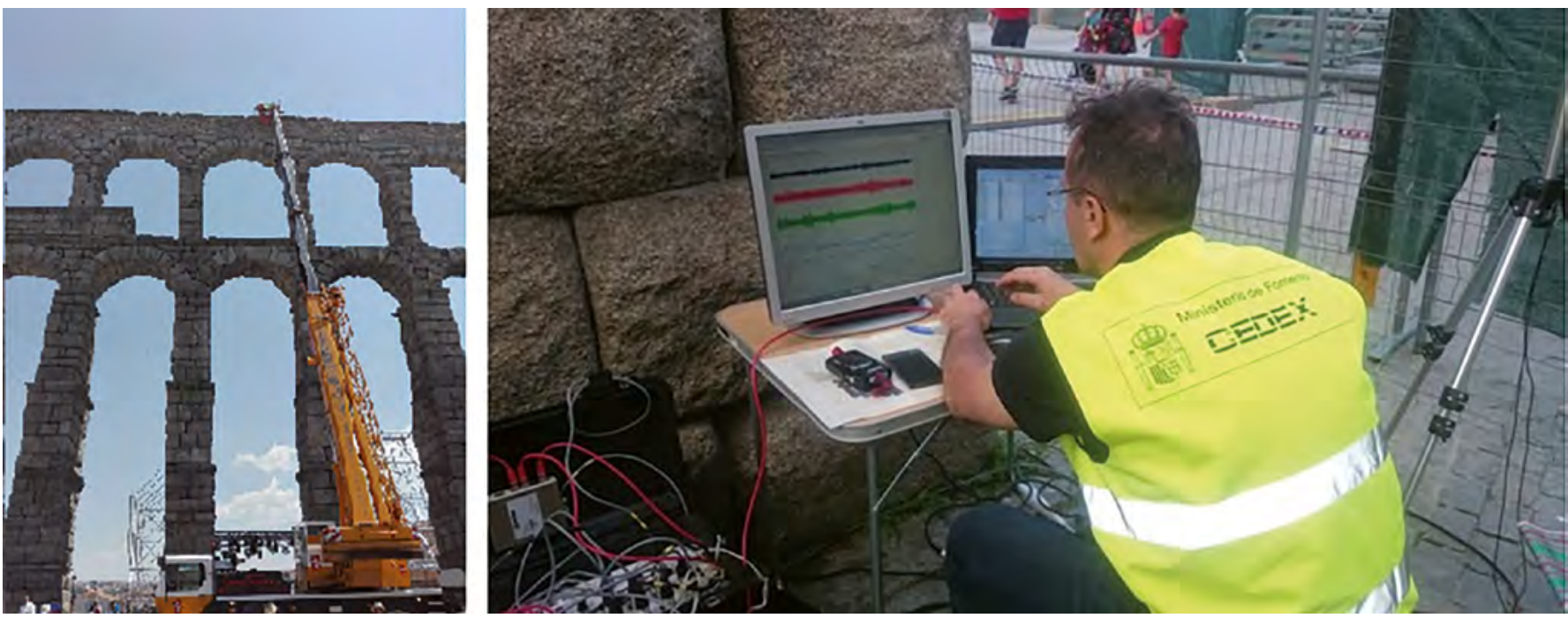

Figura 21. Estudio de vibraciones en el Acueducto de Segovia (2018).

seguimiento de la evolución del fenómeno, combinando la inspección de daños y calas con el ensayo de materiales y la realización de pruebas de carga dinámicas.

Bajo la coordinación del Laboratorio Central, entre 2014 y 2017 se llevaron a cabo cincuenta estudios multidisciplinares sobre puentes de carácter histórico de las redes nacionales de carreteras y ferrocarril, en el que participan distintos centros del CEDEX. Estos estudios, financiados con cargo a fondos del $1 \%$ Cultural, estuvieron orientados a la puesta en valor de dichas estructuras mediante su caracterización desde un punto de vista histórico, patológico-estructural, del entorno geológico-geotécnico y de las condiciones hidrológico-hidráulicas del emplazamiento ${ }^{22}$ (figura 20).

En el ámbito de la edificación se han realizado estudios de evaluación estructural de la antigua Escuela Especial de Ingenieros de Caminos del Retiro, y del edificio del Laboratorio de Alumnos; el estudio de los forjados y de la Galería de Poniente del Palacio del Infantado (figura 18); el estudio de los daños

22 Si bien no era en principio el objeto de los trabajos, gracias a estos estudios se pudieron identificar algunos puentes con problemas patológicos relevantes, e incluso en un caso fue necesario dejar fuera de servicio la estructura con carácter urgente tras la primera inspección. en la Residencia Blume del Centro de Alto Rendimiento de Madrid; el estudio de la afección estructural de las obras del Metro sobre los edificios del Complejo Dotacional "El Pilar" en San Fernando de Henares; etc.

Además de edificios y puentes se han realizado estudios sobre otro tipo de estructuras, como el depósito de Sopalmo; el depósito elevado de Torroja en la Junta de Energía Nuclear de Madrid; o la instrumentación del Acueducto de Segovia para analizar la posible afección a la estructura de los conciertos que se realizan en su entorno (figura 21). También se ha seguido trabajando en la innovación de materiales de construcción, desarrollándose varias Tesis Doctorales sobre modelos de durabilidad y el empleo de áridos reciclados para hormigones.

Por otro lado se ha seguido dando apoyo a otros órganos de la Administración en relación a los materiales de construcción y las estructuras. En este ámbito cabe destacar los trabajos de coordinación en el Capítulo de Materiales realizados para el desarrollo del futuro Código Estructural.

Siguiendo el principio de incorporar nuevas tecnologías, desde 2014, coordinados desde el Área de Estudios y Auscultación de Estructuras, se iniciaron los trabajos para la adquisición y puesta en marcha de un servicio de drones para su empleo en 

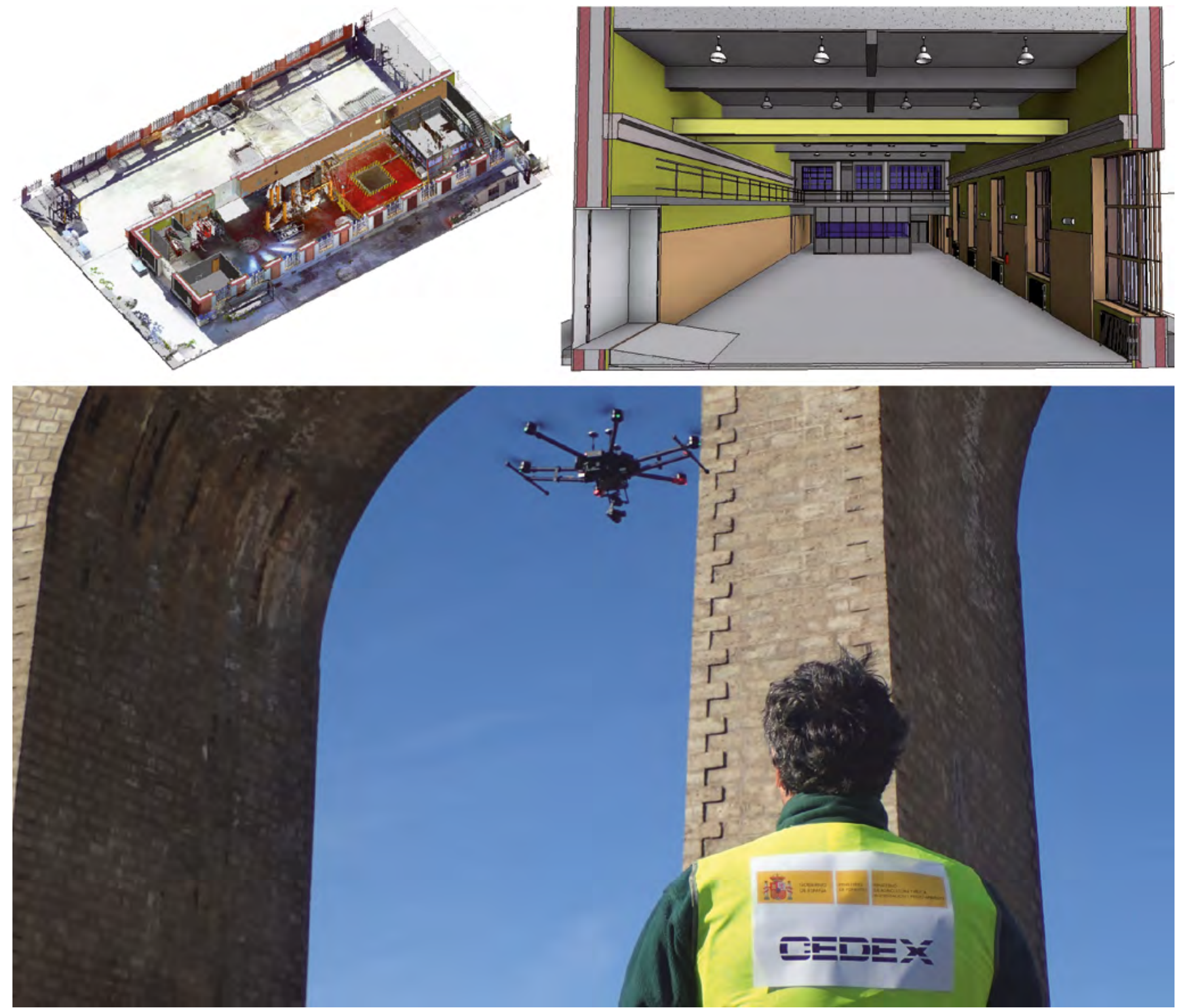

Figura 22. Operador de Drones del CEDEX (2018) y modelo BIM en construcción del edificio del Laboratorio Central (2020).

el ámbito de los distintos Centros (en particular, para la inspección de estructuras), que culminó en 2018 con la creación del Operador de Drones del CEDEX (figura 22). En los últimos tiempos también se está empezando a incorporar la tecnología BIM para registrar el estado actual de edificios, y como apoyo a la comisión BIM del Ministerio de Transportes, Movilidad y Agenda Urbana (figura 22).

10.

\section{CONCLUSIÓN}

Desde su creación, el Laboratorio Central ha tenido como principal objetivo el de incorporar nuevas tecnologías para el control de las construcciones. A tal efecto, a lo largo de sus más de cien años de historia ha ido modificando el alcance de sus trabajos, adaptando su estructura organizativa, y adquiriendo y poniendo en funcionamiento la tecnología y materiales más innovadores de cada época para su puesta a punto y transmisión a la sociedad.

Esta actividad se ha realizado de la mano de algunos de los ingenieros españoles de mayor prestigio, a los que el presente artículo trata de rendir un merecido, sincero y humilde homenaje y reconocimiento

Desde la atalaya que proporciona el tiempo transcurrido, el personal actual del Área de Estructuras del Laboratorio Central trata de subir a los hombros de sus predecesores, buscando ser dignos sucesores, al menos, de su empeño y dedicación.

\section{Agradecimientos}

La recopilación y revisión de los casi 55.000 expedientes de la actividad del Laboratorio Central desde 1899 no habría sido 


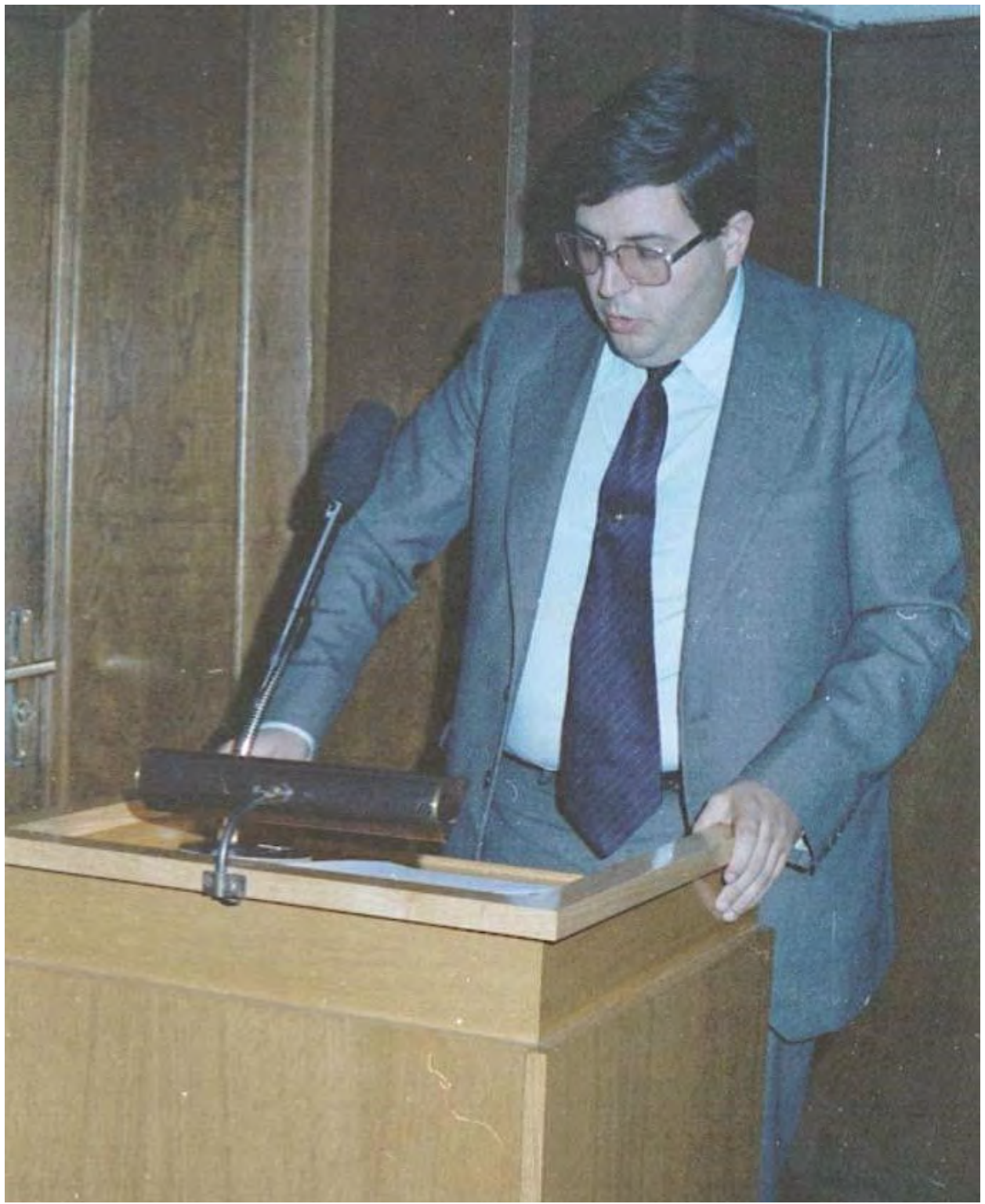

Figura 23. Luis Ortega Basagoiti. Jornada de Puertas Abiertas del Laboratorio Central (1981).

posible sin el apoyo y ayuda de María Jose Sánchez Gil, Carlos Castejón Mediano, Juan Antonio Hernández Martínez, Francisco Serrano, Rafael Astudillo Pastor, Francisco Javier Sainz de Cueto y José Manuel Gálligo Estévez.

Además, se desea reconocer de manera especial en este artículo la figura de Luis Ortega Basagoiti (figura 23) en su paso por el Laboratorio Central, en el campo concreto de la inspección y auscultación de estructuras, bajo cuya coordinación se inició una ingente actividad en este ámbito que aún hoy sigue vigente.

\section{Referencias}

[1] Graus, R et al. (2017) El hormigón armado en Cataluña (1898-1929): cuatro empresas y su relación con la arquitectura. Informes de la Construcción Vol. 69, 546, 2000 abril-junio 2017.

[2] Carpintero, I. (2013)Aproximación histórica a las condiciones de durabilidad de las estructuras en los tratados e instrucciones de hormigón en España. Monografia CEDEX n ${ }^{\circ} 121$.

[3] IETCC (1961) Instrucción H.A. 61 especial para estructuras de hormigón armado. Instituto Eduardo Torroja de la Construcción y del Cemento.

[4] Ministerio de Fomento (1998) Instrucción de Hormigón Estructural EH-98.

[5] UNE-EN (1992) Eurocódigo 2: Proyecto de estructuras de hormigón. 